\title{
Reciprocal regulation of pancreatic ductal adenocarcinoma growth and molecular subtype by HNF4 $\alpha$ and SIX1/4
}

Soledad A. Camolotto ${ }^{1}$, Veronika K. Belova ${ }^{1}$, Luke Torre-Healy ${ }^{2,3}$, Jeffery M. Vahrenkamp ${ }^{4}$,

Kristofer C. Berrett ${ }^{4}$, Hannah Conway ${ }^{1}$, Chris Stubben ${ }^{5}$, Richard Moffitt ${ }^{2}$, Jason Gertz ${ }^{4}$, Eric L. Snyder ${ }^{1}$

\author{
Affiliations \\ ${ }^{1}$ Department of Pathology and Huntsman Cancer Institute, University of Utah, Salt Lake City, UT \\ 84112, USA \\ ${ }^{2}$ Department of Biomedical Informatics \& Stony Brook Cancer Center, Stony Brook University, \\ Stony Brook, NY 11794 \\ ${ }^{3}$ Medical Scientist Training Program, Stony Brook University, Stony Brook, NY 11794 \\ ${ }^{4}$ Department of Oncological Sciences and Huntsman Cancer Institute, University of Utah, Salt \\ Lake City, UT 84112, USA \\ ${ }^{5}$ Bioinformatics Shared Resource, Huntsman Cancer Institute, University of Utah, Salt Lake City, \\ UT 84112, USA \\ Corresponding author: eric.snyder@hci.utah.edu
}

Key words: Pancreatic ductal adenocarcinoma, molecular subtype, HNF4 $\alpha$, SIX1, SIX4 


\section{Abstract}

Pancreatic ductal adenocarcinoma (PDAC) is an aggressive malignancy with a five-year survival of less than $5 \%$. Transcriptomic analysis has identified two clinically relevant molecular subtypes of PDAC: Classical and Basal-like. The Classical subtype is characterized by a more favorable prognosis and better response to chemotherapy than the Basal-like subtype. The Classical subtype also expresses higher levels of lineage specifiers that regulate endodermal differentiation, including the nuclear receptor HNF4 $\alpha$. Using in vitro and in vivo PDAC models, we show that HNF4 $\alpha$ restrains tumor growth and drives tumor cells toward an epithelial identity. Gene expression analysis from murine models and human tumors shows that HNF $4 \alpha$ activates expression of genes associated with the Classical subtype. Although HNF4 $\alpha$ loss is not sufficient for complete conversion to the Basal-like subtype gene expression profile, HNF4 $\alpha$ directly represses SIX4 and SIX1, mesodermal lineage specifiers expressed in the Basal-like subtype. Finally, HNF4 $\alpha$-negative PDAC cells rely on expression of SIX4 and SIX1 for proliferation in vitro and in vivo. Overall, our data show that HNF4 $\alpha$ regulates the growth and molecular subtype of PDAC by multiple mechanisms, including activation of the Classical gene expression program and repression of SIX4 and SIX1, which may represent novel dependencies of the Basal-like subtype.

\section{Introduction}

Pancreatic ductal adenocarcinoma (PDAC) is an extremely aggressive malignancy with a fiveyear survival of less than 5\% (1). Several recent genome-wide analyses of PDAC have identified discrete molecular subtypes of this disease (2-4). Most recently, the Cancer Genome Atlas Research Network reported an integrated genomic, transcriptomic and proteomic analysis of an additional 150 PDAC samples (5). This integrative analysis found significant overlap between the different subtypes, ultimately suggesting that PDAC can fundamentally be classified into two 
major subtypes based on cancer cell autonomous properties: Classical and Basal-like. These subtypes appear to be clinically relevant because the Classical subtype is associated with a better overall survival and better response to first line chemotherapy than the Basal-like subtype $(2,4,6)$.

One major difference between these two subtypes is the relative levels of endodermal lineage specifiers, i.e. transcription factors that regulate development and differentiation of endodermallyderived tissues. The Classical subtype expresses high levels of these genes, including HNF4A, GATA6, FOXA2, FOXA3, and others (2). Histopathologic and gene expression analysis have shown that pancreatic intraepithelial neoplasia (PanIN, the most common non-invasive precursor lesion of PDAC) and well-differentiated PDACs exhibit a cellular identity that is distinct from the normal pancreas and is characterized by upregulation of transcripts highly expressed in the foregut. This suggests pancreatic neoplasia initially adopts a cellular identity resembling the tissue from which the pancreas originated during development $(7,8)$. Moreover, these correlations raise the question of whether specific endodermal lineage specifiers are not merely markers of discrete subtypes, but might directly regulate molecular subtype, malignant potential and therapeutic response in PDAC.

The nuclear receptor superfamily member HNF4 $\alpha$ is a master regulator of epithelial differentiation in multiple tissues, including the gastrointestinal (GI) tract and the liver (9-12). HNF4 $\alpha$ also regulates a variety of biological processes that impact tumor progression, including proliferation (13), metabolism (14), and inflammation (15). Consistent with genomics studies mentioned above, HNF4 $\alpha$ protein is detectable in human pancreatic neoplasia, and its levels correlate with tumor differentiation state (16). Immunohistochemistry (IHC) on tissue sections has shown that PanIN lesions and well-differentiated PDAC express higher levels of HNF4 $\alpha$ than the normal pancreas, whereas HNF4 $\alpha$ is downregulated in poorly differentiated PDAC. Nine proteins that 
are part of an HNF4 $\alpha$-regulated network were identified by proteomic analysis of plasma from mice with PanIN lesions $(16,17)$, suggesting that HNF4 $\alpha$ is functionally active in early pancreatic neoplasia.

In previous work, we have shown that HNF4 $\alpha$ plays a critical role in a mouse model of invasive mucinous adenocarcinoma of the lung (18), a subtype of lung cancer that expresses many of the same foregut markers observed in the Classical subtype of PDAC. Based on these data and the differential expression of HNF4 $\alpha$ between the Classical and Basal-like subtypes of human PDAC, we sought to determine whether HNF4 $\alpha$ also plays a functional role in this disease. We found that $\mathrm{HNF} 4 \alpha$ is a major activator of the Classical gene expression program and restrains PDAC growth in multiple models of the disease. Although HNF4 $\alpha$ loss is not sufficient for complete subtype switching, HNF4 $\alpha$ represses the expression of SIX4 and SIX1, two mesodermal lineage specifiers highly expressed in the Basal-like subtype of PDAC.

\section{Results}

Hnf4a deletion accelerates tumorigenesis in a mouse model of pancreatic ductal adenocarcinoma

To dissect the role of HNF4 $\alpha$ in PDAC, we first used a mouse model of pancreatic neoplasia that closely mimics the human disease. This model employs the Pdx1-Cre transgene to activate expression of $\mathrm{KRAS}^{\mathrm{G} 12 \mathrm{D}}$ from its endogenous locus during pancreatic development, which thereby induces neoplasia that closely models human PanIN and PDAC $(19,20)$. Greater than $90 \%$ of human PanINs and PDACs contain mutations that result in constitutive activation of the KRAS oncogene, which is believed to be the initiating event in most cases (21). KRAS is a small GTPase 
that interacts with receptor tyrosine kinases at the plasma membrane to transduce growth factorinduced signals to several intracellular effectors (22).

Pdx1-Cre; Kras ${ }^{L S L-G 12 D}$ mice develop acinar to ductal metaplasia (ADM) and murine PanIN (mPanIN) lesions with complete penetrance, and these in situ lesions occasionally progress to invasive cancer in older mice. To accelerate cancer progression, a conditional allele of the p53 tumor suppressor is incorporated into the model. Excision of one allele of $p 53\left(P d x 1-C r e ; K r a s^{L S L-}\right.$ G12D; $p 53^{F /+}$ mice) accelerates neoplastic progression to invasive and metastatic adenocarcinomas, which often develop in mice 3-6 months of age (19). We initially evaluated a cohort of control mice $\left(P d x 1-C r e ; K s^{L S L-G 12 D} ; p 53^{F /+} ; H_{n f 4 a^{+/+}}\right)$at 12 weeks of age $(n=17$, Figure 1A-C). As expected from published reports (16), HNF4 $\alpha$ was robustly expressed in preinvasive lesion (ADM and mPanIN) arising in these mice (Figure 1A, left). Careful evaluation of HNF4 $\alpha$ levels in the subset of mice harboring PDAC $(n=7)$ showed that $3 / 7$ tumors were diffusely $(>90 \%)$ HNF4 $\alpha$-positive. In contrast, one tumor contained a mix of HNF4 $\alpha$-positive and negative cells and 3/7 tumors were completely HNF4 $\alpha$-negative, despite the presence of adjacent HNF4 $\alpha$-positive mPanIN (Figure S1A). HNF4 $\alpha$-positive and mixed tumors exhibited a range of differentiation states (including well, moderately and poorly differentiated), whereas the three HNF4 $\alpha$-negative tumors were poorly differentiated. Taken together, these data shown that HNF4 $\alpha$ is expressed at higher levels in early pancreatic neoplasia than normal pancreas, but that HNF4 $\alpha$ can also be stochastically downregulated during tumor progression. 
Figure 1, Camolotto et al.

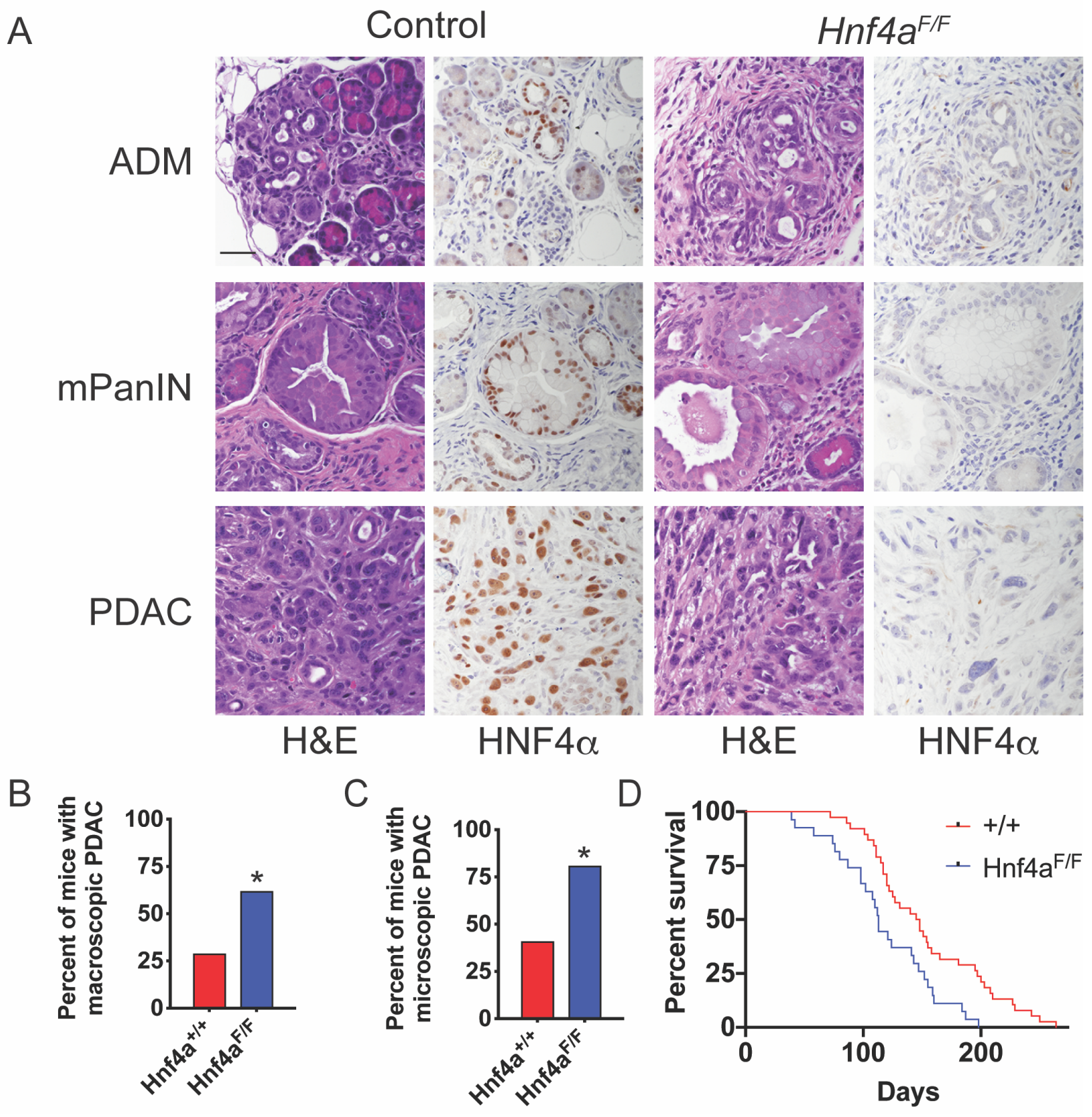


Figure 1. Hnf4a deletion accelerates tumorigenesis in a mouse model of pancreatic ductal adenocarcinoma.

A. H\&E and IHC for HNF4 $\alpha$ on pancreatic neoplasia from Kras ${ }^{L S L-G 12 D /+;} p 53^{F /+} ; P d x 1-C r e ; H n f 4 a^{F / F}$ mice and $\mathrm{Hnf}_{4 \mathrm{a}^{+/+}}$controls at 12 weeks of age. Scale bar: 50 microns. ADM: acinar to ductal metaplasia. mPanIN: Pancreatic intraepithelial neoplasia.

B-C. Percentage of Kras ${ }^{L S L-G 12 D /+;} p 53^{F /+} ;$ Pdx1-Cre; Hnf4a $a^{F / F}$ mice $(\mathrm{n}=21)$ and $\mathrm{Hnf4a}^{+/+}$controls $(n=17)$ with macroscopic $(B)$ and microscopic $(C)$ PDAC at 12 weeks of age. ${ }^{*} p<0.05$, Chi-Square.

D. Survival curve of $\mathrm{Kras}^{L S L-G 12 D /+;} p 53^{F /+} ; \mathrm{Pdx1}$-Cre; Hnf4a $a^{F / F}$ mice $(\mathrm{n}=27)$ and $\mathrm{Hnf} 4 \mathrm{a}^{+/+}$controls $(n=38) . p=0.0027$, Log-rank. 
Twelve isoforms of HNF4 $\alpha$ have been identified to date (23), which arise as a result of transcription from two different promoters (P1 and P2) as well as alternative exon splicing. The $\mathrm{P} 1$ and P2 promoters are activated in a tissue-specific manner, and P1 and P2 isoforms have distinct roles in tumorigenesis $(24,25)$. Using monoclonal antibodies specific for the P1 and P2 isoforms, we found that HNF4 $\alpha$-positive neoplasia predominantly expressed the P2 isoform in this model (Figure S1B).

In contrast to control mice, all neoplasia (ADM, mPanIN and PDAC) in $H n f 4 a^{F / F}$ mice at 12 weeks was HNF4 $\alpha$-negative ( $n=21$, Figure 1A, right). We found no evidence of incomplete recombinants, which can be observed in the Cre-Lox system when there is selection for retention of a conditional allele. Although Hnf4a deletion had no obvious effect on the morphology or quantity of preinvasive lesions, it significantly increased the proportion of mice with either macroscopic PDAC (Figure 1B) or microscopic PDAC (Figure 1C) at 12 weeks of age.

In a separate survival analysis, we found that Hnf4a deletion significantly reduces survival in this model (median survival 113 days in $\mathrm{Hnf}_{4} \mathrm{a}^{\mathrm{F} / \mathrm{F}}$ mice vs. 147 days in $\mathrm{Hnf4a}^{+/+}$mice, Figure 1D). Consistent with our analysis of tumors in 12-week old mice, a subset of tumors in $\mathrm{HnfHa}^{+/+}$mice exhibited stochastic HNF4 $\alpha$ downregulation, whereas tumors in $H n f 4 a^{F / F}$ mice were consistently HNF4 $\alpha$-negative. Specifically, 9/20 tumors available for evaluation in $\mathrm{Hnf}_{4} \mathrm{a}^{+/+}$mice were HNF4 $\alpha$ mixed and 2/20 were HNF4 $\alpha$-negative (all of which were included in the overall survival anlaysis). Most tumors in the survival analysis harbored both moderately and poorly differentiated components, although a minority were entirely poorly differentiated $\left(2 / 20\right.$ in $\mathrm{Hnf}_{4} \mathrm{a}^{+/+}$mice and 4/12 evaluated in $H n f 4 a^{F / F}$ mice). Almost all survival mice $\left(18 / 20 \mathrm{Hnf}^{+} \mathrm{a}^{+/+}\right.$and $11 / 12 \mathrm{Hnf}^{\mathrm{F}} \mathrm{a}^{\mathrm{F} F}$ 
mice) harbored metastases to sites such as liver, kidney, peritoneal lymph nodes, diaphragm and the lung.

In the absence of p53 mutations, Pdx1-Cre; Kras ${ }^{L S L-G 12 D}$ mice develop ADM and PanIN with high penetrance, but these lesions rarely progress to PDAC (19). To determine whether Hnf4a deletion would be sufficient for PDAC development, we aged a cohort of Pdx1-Cre; Kras ${ }^{\text {LSL-G12D; }}$ $\mathrm{Hnf}_{4} \mathrm{a}^{\mathrm{F} / \mathrm{F}}$ mice (and $\mathrm{Hnf4a}^{+/+}$controls, $\mathrm{n}=4-8$ mice/group at each timepoint) and analyzed their pancreata at 6 and 9 months of age. We observed ADM and PanIN in nearly all mice of both genotypes, but no evidence of PDAC, despite the lack of HNF4 $\alpha$ expression in Hnf4a ${ }^{F / F}$ mice (Figure S1C).

Taken together, these data show that HNF4 $\alpha$ restrains the growth of pancreatic neoplasia in this model system, predominantly at the stage of invasive PDAC. These results are also consistent with the biology of human PDAC, specifically the observation that Basal-like (HNF4 $\alpha$-low) tumors confer a worse prognosis, but that both HNF4 $\alpha$-positive and HNF4 $\alpha$-negative disease are highly lethal $(2,4,6)$.

\section{HNF4 $\alpha$ reconstitution impairs PDAC growth and imposes epithelial differentiation in vitro} and in vivo

To more precisely define the role of HNF4 $\alpha$ in PDAC, we developed additional systems to manipulate its expression in established neoplasia. First, we generated two cell lines (HC800 and HC569) from PDAC arising in Pdx1-Cre; Kras ${ }^{L S L-G 12 D} ; p 53^{F /+} ; H_{n f 4 a} a^{F / F}$ mice using standard two-dimensional (2D) culture conditions. We then stably transduced these cell lines with lentivirus enabling doxycycline (dox) induction of the HNF4 $\alpha 8$ isoform, a major product of the P2 promoter. 
We used a P2 isoform for reconstitution studies because only HNF4 $\alpha-\mathrm{P} 2$ is expressed in the autochthonous model (Figure S1B). We then obtained single cell clones that exhibited optimal dox induction of HA-tagged HNF4 $\alpha$ (Figure S2A) and subjected them to proliferation analysis. Exogenous HNF4 $\alpha$ significantly inhibited the proliferation of both cell lines in vitro (Figure $2 \mathrm{~A}$ ) with no obvious induction of apoptosis as assessed by cleaved caspase-3 (Figure S2B). HC800 cells expressing exogenous HNF4 $\alpha$ exhibited a more epithelial morphology in vitro (Figure S2C), suggesting that $\mathrm{HNF} 4 \alpha$ reconstitution also modulated their differentiation state.

To test the effect of HNF4 $\alpha$ restoration in vivo, we first injected cells subcutaneously into mice fed control or dox-containing chow to induce HNF4 $\alpha$. HNF4 $\alpha$ was robustly expressed in tumors of mice on dox chow (Figure S2D). Exogenous HNF4 $\alpha$ significantly inhibited tumor growth of both murine PDAC cell lines as assessed by both volume and mass (Figure 2B), and this was accompanied by a significantly lower proliferation rate than controls (Figure 2C). To better recapitulate the biology of PDAC, we also evaluated the growth of $\mathrm{HC} 800$ cells in the peritoneal space, which is one of the first sites of extra-pancreatic dissemination as PDAC progresses. We found that these cells grew readily in the peritoneum, forming numerous macroscopic nodules on the serosal surface of intraperitoneal organs. In a formal survival study, dox-mediated induction of $\mathrm{HNF} 4 \alpha$ reduced tumor burden at 20 days post injection (Figure 2D) and significantly increased overall survival (Figure 2E). 
Figure 2, Camolotto et al.

A

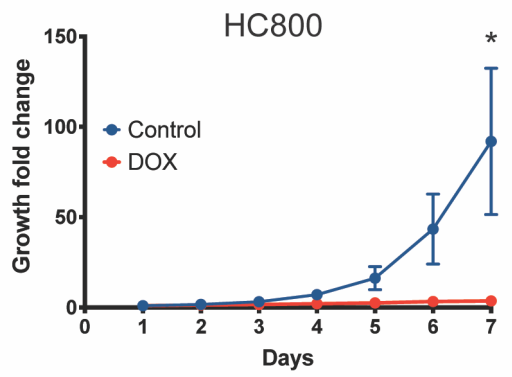

B

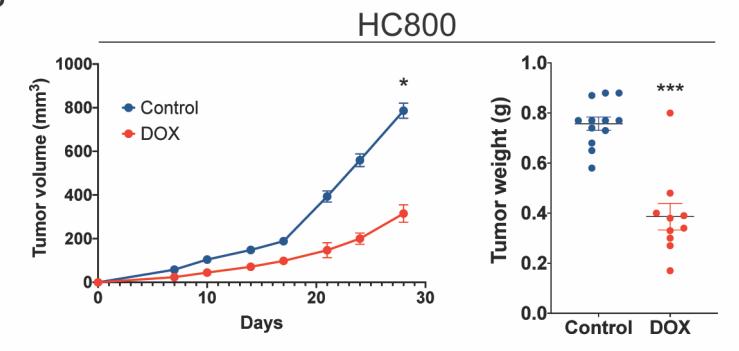

PDX220
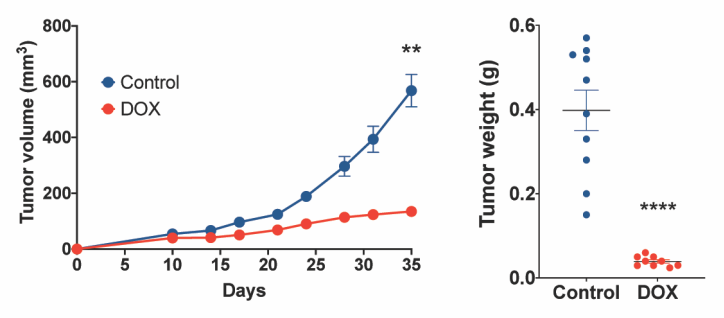
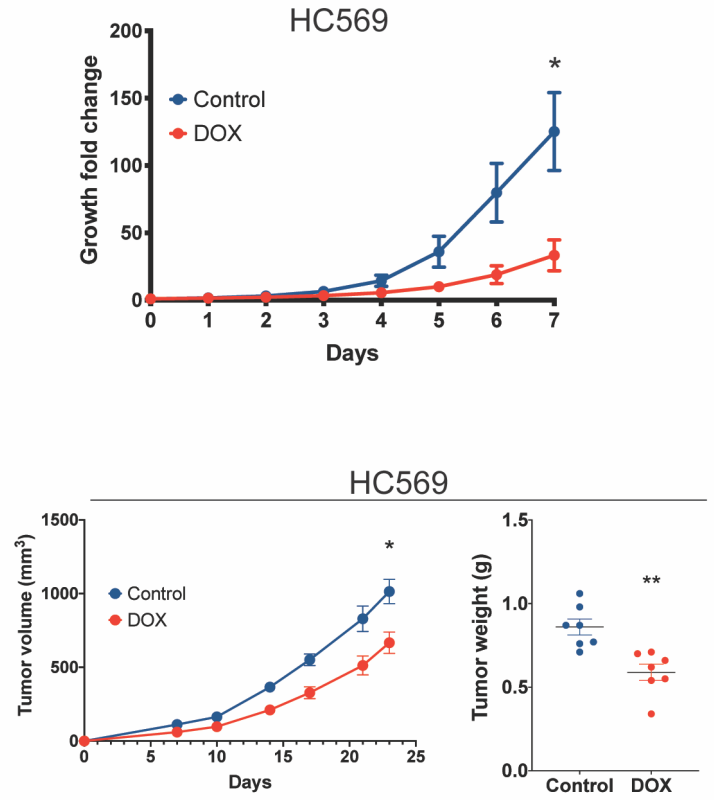

C

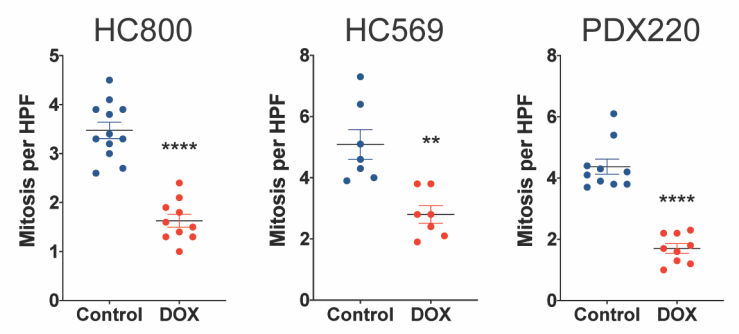

D

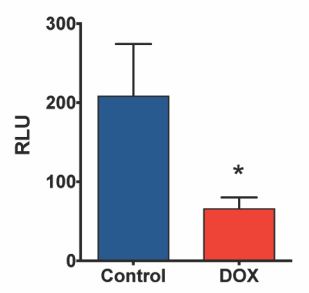

E

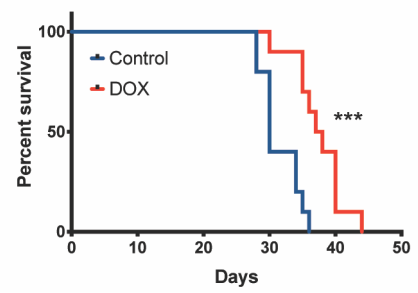

F

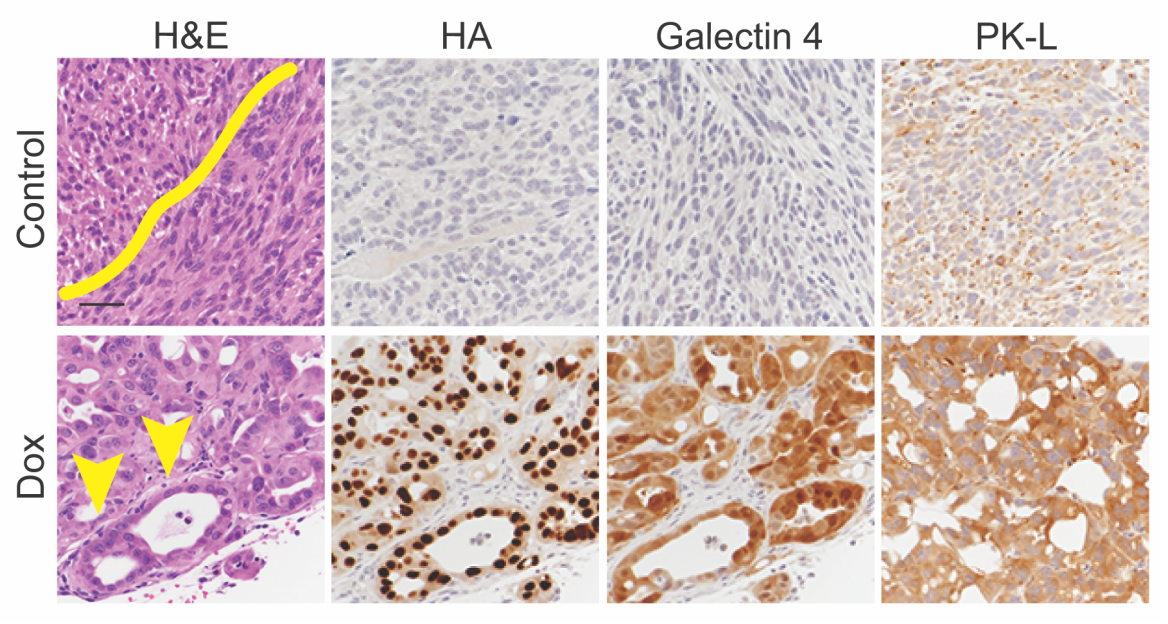


Figure 2. Restoration of HNF4 $\alpha$ expression inhibits proliferation and induces epithelial differentiation (mesenchymal to epithelial transition) in PDAC.

A. Quantitation of proliferation in murine (HC800 and HC569) PDAC cells stably expressing TREHNF4 $\alpha 8$ cells the presence or absence of doxycycline ( $n=3$ biological replicates, $2-3$ independent experiments). Primary murine PDAC cell lines were single cell cloned. Graph represents mean +/- SEM. $p=0.03$, Wilcoxon.

B-C. HC800, HC569, and PDX220 cells stably transduced with TRE-HNF4 $\alpha 8$ were injected subcutaneously into NOD-SCID-gamma chain deficient (NSG) mice. Mice were fed with doxycycline chow (red) or regular chow (blue) starting 1 week before transplantation. B. Flank tumor volume and mass are shown. C. Flank tumor growth was assessed by measuring mitoses per high power field (HPF). Data represented as mean \pm SEM. ${ }^{*} p<0.05 ;{ }^{* *} p<0.01 ;{ }^{* *} p<0.001$ by Wilcoxon test (for tumor volume) or Mann-Whitney test (for tumor mass and mitoses).

D-F. HC800-TRE-HNF4 $\alpha 8$ cells were injected intraperitoneally into NSG mice. Mice received either doxycycline chow or control chow starting 1 week prior to cell injection $(n=10$ mice per group). Tumor cells were stably transduced with luciferase expression vector prior to injection. D. Quantitation of relative light units (RLU) in each group 20 days post flank injections. Graph shows fold change in RLU normalized to basal measurements. Data represented as mean \pm SEM. ${ }^{*} p<0.05$ by $t$ test. E. Survival analysis. ${ }^{* * *} p<0.001$ by log-rank test. F. Representative H\&E and IHC for HA-tagged HNF4 $\alpha$ and target genes in intraperitoneal tumors from each group. Yellow line demarcates two fascicles of tumor cells oriented in opposite directions. Yellow arrows mark glandular differentiation. Scale bar: 100 microns. 
In addition to restraining proliferation, $\mathrm{HNF} 4 \alpha$ had a clear impact on differentiation state in vivo, shifting tumors toward a more well differentiated epithelial morphology (Figures 2F and S2D). This was particularly striking in the HC800 cell line. Control HC800 tumors had a uniform morphology composed of spindled cells growing in a fascicular pattern that is characteristic of a sarcomatoid or quasi-mesenchymal differentiation state (Figure $2 \mathrm{~F}$, top row). IHC for the HA tag confirmed that exogenous HNF4 $\alpha$ was not expressed in these tumors. In contrast, tumors growing in mice fed dox chow adopted the morphology of a moderately to poorly differentiated adenocarcinoma, with a glandular rather than fascicular growth pattern and cells that were round rather than spindled. These tumors expressed uniformly high levels of HA-tagged HNF4 $\alpha$, its target PK-L, and Galectin 4, a marker of foregut differentiation (Figure 2F, bottom row).

To further evaluate these results in human PDAC cells, we stably transduced a human PDAC cell line derived from the xenograft PDX220 (26) with lentivirus encoding dox-inducible HNF4a8 and tested the effect of HNF4a induction on subcutaneous tumor growth. Control PDX 220 tumors contain a mixture of HNF4 $\alpha$-positive and -negative cells (Figure S2E). Interestingly, HNF4 $\alpha$ positive cells are a moderately differentiated adenocarcinoma, whereas the HNF4 $\alpha$-negative cells consist of squamous cell carcinoma that expresses $\Delta \mathrm{Np63}$, a marker of squamous differentiation (Figure S2E-F). Exogenous HNF4 $\alpha$ substantially inhibited overall PDX220 tumor growth and cellular proliferation (Figure 2B-C). Moreover, tumors arising in dox-treated mice consisted entirely of adenocarcinoma that was better differentiated than control tumors (Figure S2F), showing that HNF4 $\alpha$ not only suppressed squamous differentiation in this model but also modulated the differentiation state of the adenocarcinoma component.

Taken together, these results show that restoring HNF4 $\alpha$ function can induce major changes in differentiation state and inhibit PDAC growth both in vitro and in vivo. 


\section{Hnf4a deletion in PDAC organoids alters differentiation and three dimensional growth pattern in vivo}

Given that HNF4 $\alpha$ is often stochastically downregulated during PDAC progression, we next developed a system that would enable us to inactivate endogenous Hnf4a in established murine pancreatic neoplasia. In mouse models of lung cancer, we have previously used a sequential recombinase strategy to delete genes in established tumors (18). Merging this approach with pancreatic organoid culture systems (27), we established PDAC organoids by enzymatically dissociating pancreata from Kras ${ }^{\text {FSF-G12D/+; }} p 53^{\text {Frt/Frt; }}$ Rosa26 $6^{F S F-C r e E R T 2} ; H n f 4 a^{F / F}$ mice. We then treated these cells in vitro with adenovirus expressing the FlpO recombinase, which activated transcription of oncogenic KRAS ${ }^{G 12 D}$ and inactivated p53, leading to the emergence of HNF4 $\alpha$ positive PDAC organoids (Figure $3 \mathrm{~A}$ and S3A). FlpO also activated transcription of Cre ${ }^{\text {ERT2 }}$ from the Rosa26 locus. This enabled us to treat these cultures with 4-hydroxy-tamoxifen (4-OHT), thereby driving Cre ${ }^{\mathrm{ERT} 2}$ into the nucleus where it excised the respective conditional alleles. IHC and qRT-PCR demonstrated loss of HNF4 $\alpha$ as well as its target Pklr in four independently derived organoid cultures (Figure S3A-B).

We next asked whether loss of HNF4 $\alpha$ would affect the growth and differentiation state of these organoid lines in vivo. We injected two lines (SC1853 and SC1693) subcutaneously into NOD/SCID-gamma chain deficient (NSG) mice. In mice treated with control chow, both lines formed macroscopic, fluid filled cysts (Figure 3B and S3C). These cysts were predominantly lined by a single layer of HNF4 $\alpha$-positive columnar epithelial cells (Figure 3C and S3D, top row). We also identified small areas of well-differentiated adenocarcinoma (Figure 3C and S3D, bottom row) within the walls of these cystic structures. 
Figure 3, Camolotto et al.

A

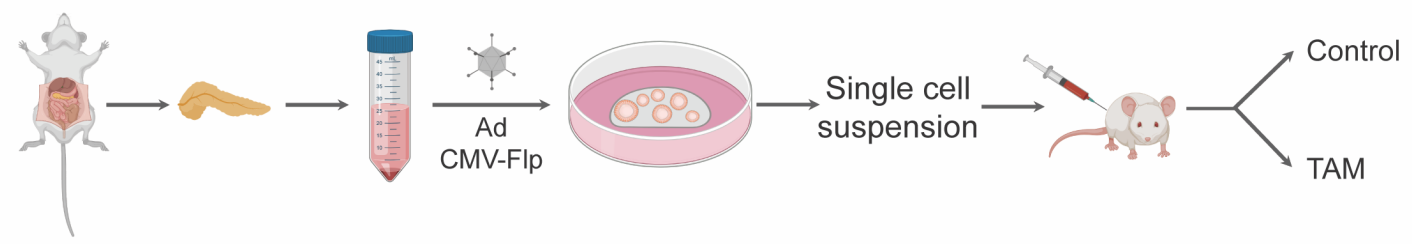

B

C

HNF4 $\alpha$
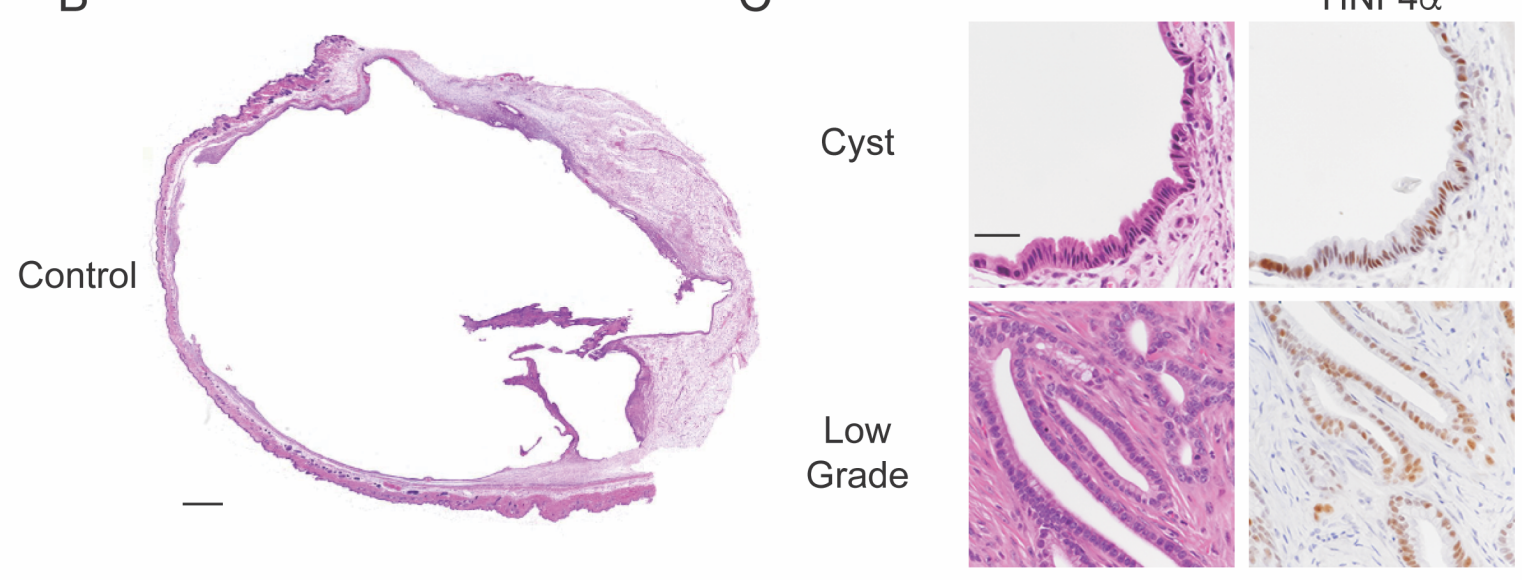

D

E

HNF4 $\alpha$

TAM

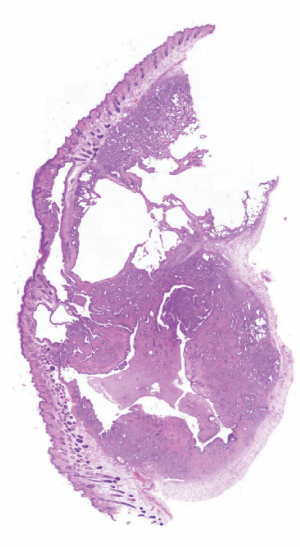

Intermediate

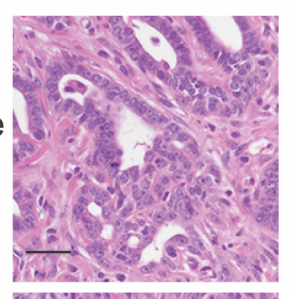

High

Grade

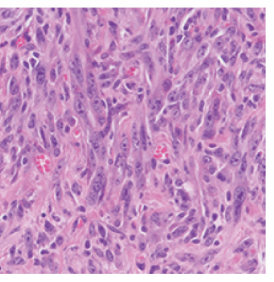


Figure 3. Hnf4a deletion alters differentiation and three dimensional growth in murine organoid models of PDAC.

A. Schematic of organoid derivation from pancreata of Kras ${ }^{\text {FSF-G12D/+; }} p 3^{\text {Frt/Frt; }}$ Rosa26 $6^{\text {FSF-CreERT2; }}$ Hnf4a $a^{F / F}$ mice.

B-E. H\&E and IHC analysis of a single cell suspension of SC1853 organoids injected subcutaneously into NSG mice. Mice were fed control (B-C) or tamoxifen (D-E) chow starting at 1 week prior to organoid implantation. Tumors were analyzed at 6 weeks post injection. B and D: scanning magnification, scale bar: $1 \mathrm{~mm}$. C and E: High power images, scale bar: 100 microns. 
In contrast, tumors that grew in mice on tamoxifen chow exhibited a completely distinct threedimensional growth pattern and differentiation state. These tumors consisted of a mixture of invasive adenocarcinoma and microscopic cystic structures (Figure 3D and S3E). Moreover, the adenocarcinoma component was less well differentiated than control tumors. Hnf4a-deleted tumors from both organoid lines exhibited areas of moderately differentiated (intermediate grade) HNF4 $\alpha$-negative adenocarcinoma (Figure 3E and S3F). Moreover, Hnf4a deletion in tumors of the SC1853 organoid was sufficient to induce areas of high grade, poorly differentiated adenocarcinoma (Figure 3E, bottom row).

\section{HNF4 $\alpha$ activates the Classical gene expression program in PDAC}

Given its impact on the growth and differentiation state in multiple models of PDAC, we sought to formally test the hypothesis that HNF4 $\alpha$ regulates molecular subtype in PDAC. As expected from prior studies $(2,5)$, we observed a robust correlation $(r=0.71, p=9.1 e-13)$ between HNF4A expression and Classical Score in high purity tumors from the TCGA-PAAD data (Figure 4A, left). In comparison, HNF4A levels and Basal-like score were weakly negatively correlated $(r=-0.48$, $p=1.4 \mathrm{e}-5$ ) in this dataset (Figure 4A, right). We also evaluated the relationship between HNF4A levels and survival in high purity tumors (>30\% cellularity) from two independent datasets $(2,5)$ by either splitting cases around median HNF4A expression or into upper and lower quartiles of HNF4A expression (Figure S4A). In the PACA-AU dataset, patients with tumors in the upper quartile of HNF4A expression survived significantly longer than in the lowest quartile $(p=0.012)$, consistent with known survival differences between the Classical and Basal-like subtypes. Other analyses showed a similar (though non-significant) trend. Interestingly, in the TCGA dataset, a 
subset of patients with HNF4A-high tumors were still alive at the time of analysis, whereas none of the patients in the HNF4A-low cohort survived beyond the last recorded time point (2000 days).

Next we performed gene expression analysis on autochthonous murine PDAC tumors from $\mathrm{Hnf}_{4} \mathrm{a}^{+/+}$and $\mathrm{Hnf} 4 \mathrm{a}^{\mathrm{F} / \mathrm{F}}$ mice ( $\mathrm{n}=6$ per genotype), using IHC to exclude any tumors from wild type mice that had stochastically downregulated HNF4 $\alpha$. We identified a total of 597 differentially expressed genes in this analysis (Supplemental Table 1), including known HNF4 $\alpha$ targets in normal tissue such as Pklr and Apob. To determine whether Hnf4a deletion altered molecular subtype of these tumors, we assigned a Classical and Basal-like score to each dataset based on mouse homologues of the scores described in (4). The Classical score of Hnf4a-deleted tumors was significantly lower than $\mathrm{Hnf4a}^{+/+}$tumors (Figure 4B-C, left panels). In contrast, the Basal-like score was not significantly different between the two groups, although there was a slight trend toward a higher score in the Hnf4a-deleted tumors. Evaluation of lineage specifiers that regulate endodermal differentiation (Figure S4B) showed that some appear to be HNF4 $\alpha$ targets in this model (e.g., HNF1 $\alpha$, FoxA3 and CDX2), whereas others are not significantly affected by Hnf4a deletion, suggesting they are situated adjacent to or upstream of HNF4a in the hierarchy of lineage specifiers specifying the Classical subtype of PDAC. 


\section{Camolotto et al., Figure 4}

A

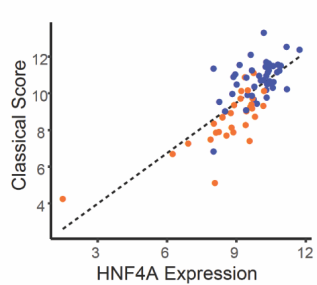

B

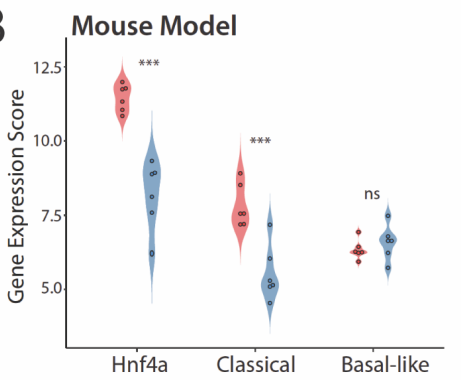

C

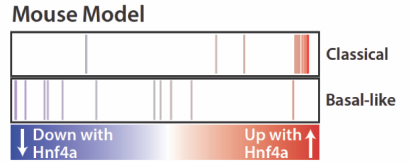

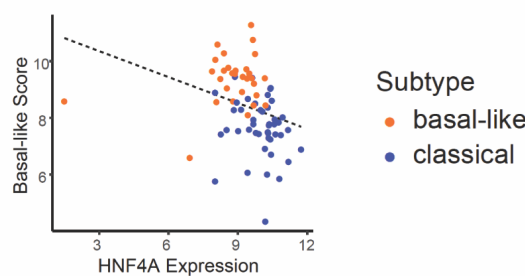
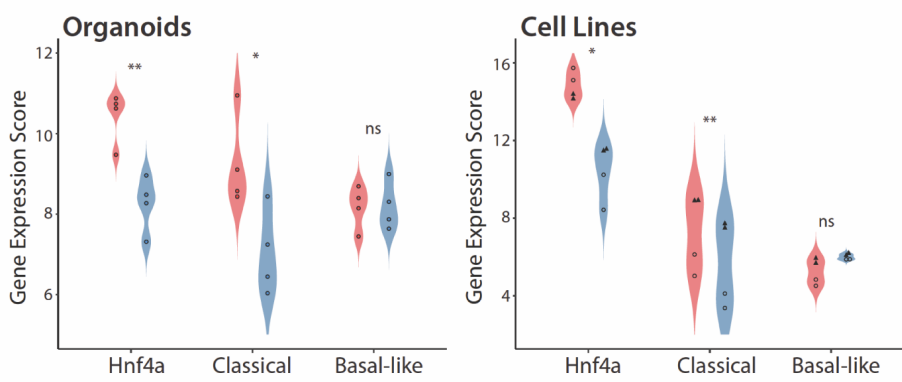

Organoids

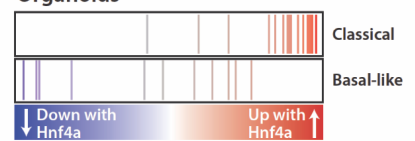

Cell Lines

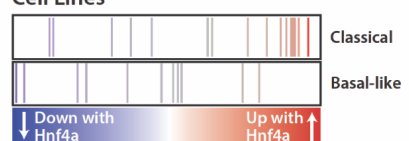




\section{Figure 4. HNF4 $\alpha$ regulates PDAC molecular subtype.}

A. Correlation between HNF4A expression in patients with Classical Score $(r=0.71, p=9.1 e-13)$ and Basal-like score $(r=-0.48, p=1.4 e-5)$ in TCGA-PAAD data.

B. Scores were generated for Basal-like and Classical subtype expression from RNA-seq data. Each point represents one sample, and samples were grouped by their HNF4 $\alpha$ status. Subtype scores were calculated using mouse homologs of the established Moffitt et al. signatures. pvalues obtained via t-test (paired for cell lines). ${ }^{* * *} p<0.001,{ }^{* *} p<0.01,{ }^{*} p<0.05$.

C. Gene Set Enrichment Analysis was performed comparing the HNF4 $\alpha$-positive samples to the HNF4 $\alpha$-negative samples, and their respective Signal-to-Noise Ratios (SNR) are plotted. Red indicates that expression of the gene increases with HNF4 $\alpha$ expression, while blue indicates expression decreases. 
Using Illumina Correlation Engine, we performed pathway analysis on genes differentially expressed between HNF4 $\alpha$-positive and HNF4 $\alpha$-negative autochthonous tumors (Supplemental Table 2). GO terms and pathways associated with epithelial differentiation (e.g., "cell-cell adhesion") and other known functions of HNF4 $\alpha$ (e.g., "Maturity onset diabetes of the young" and various metabolic pathways) were significantly enriched in HNF4 $\alpha$-positive tumors. In contrast, GO terms associated with mesodermal differentiation (e.g., "regulation of neurogenesis" and "skeletal system development") were significantly enriched in HNF4 $\alpha$-negative tumors. Consistent with these results, the Body Atlas analysis tool showed that the gene expression profile of HNF4 $\alpha$-positive tumors correlates highly with endodermally derived tissues, such as the $\mathrm{Gl}$ tract, whereas HNF4 $\alpha$-negative tumors correlate with mesodermally derived tissues found in the central nervous system and cardiovascular system (Figure S4C and Supplemental Table 2).

To expand upon these observations and determine whether HNF4 $\alpha$ can regulate PDAC identity in vitro in a cell-autonomous manner, we performed gene expression analysis on the previously described cell lines and organoids isogenic for HNF4 $\alpha$ expression (Supplemental Tables 3 and 4). In both cell line systems, HNF4 $\alpha$-positive cells had a significantly higher Classical score than HNF4 $\alpha$-negative cells (Figure 4B-C, middle and right hand panels). In contrast, the Basal-like score did not significantly change with HNF4 $\alpha$-expression. Intersection of all three datasets identified a total of 49 genes that were upregulated by HNF4 $\alpha$ in all model systems (Figure S4D and Supplemental Table 5). Although few genes were downregulated by HNF4 $\alpha$ in all model systems, primarily due to lack of overlap with the organoid dataset, we identified 65 genes downregulated by HNF4 $\alpha$ both in vivo and in 2D cell lines (Figure S4D and Supplemental Table 5). 
Taken together, these data show that HNF4 $\alpha$ is a critical regulator of cellular identity and molecular subtype in multiple models of PDAC. Specifically, HNF4 $\alpha$ is required for activation of the complete Classical gene expression program. Although loss of HNF4 $\alpha$ is not sufficient for full conversion to a Basal-like state, a subset of genes associated with the Basal-like subtype appear to be repressed by HNF4 $\alpha$.

\section{Analysis of HNF4 $\alpha$-chromatin interactions in PDAC}

In order to identify direct HNF4 $\alpha$ target genes and investigate the impact of HNF4 $\alpha$ on chromatin accessibility, we performed ChIP-seq with an antibody that recognizes the HA-tagged HNF4 $\alpha$ as well as ATAC-Seq in HC800 and HC569 cells. For both assays, cells were treated with dox or control media for one week prior to chromatin analysis. We identified 18,419 and 15,593 reproducibly significant peaks by HA ChIP-seq in HC569 and HC800 dox-treated cells, respectively. We found that $84.4 \%$ of all sites were present in both cell lines, showing a strong degree of concordance in HNF4 $\alpha$ binding (Figure 5A and S5A). As expected, motifs bound by HNF4 $\alpha$ and HNF4 $\gamma$ were the most significantly enriched in ChIP-seq peaks (Figure S5B). Examination of HNF4 $\alpha$ binding distribution showed that $45 \%$ of peaks localized to promoters, whereas $13 \%$ were found in distal regions (Figure 5B). We corroborated HNF4 $\alpha$ occupancy in relevant and well-known direct target genes such as Pklr, Lgals4, and Hnf1a (Figure 5C). To explore the connection between binding sites and gene expression, we intersected our ChIP-seq and RNA-seq datasets. We observed $69 \%$ of the differentially expressed genes displaying binding sites for HNF4 $\alpha$ (Figure 5D). For those genes bound by HNF4 $\alpha$, we found that $61 \%$ were upregulated and $39 \%$ downregulated, indicating that HNF4 $\alpha$ preferentially acts as a transcriptional activator in this context. 


\section{Camolotto et al., Figure 5}

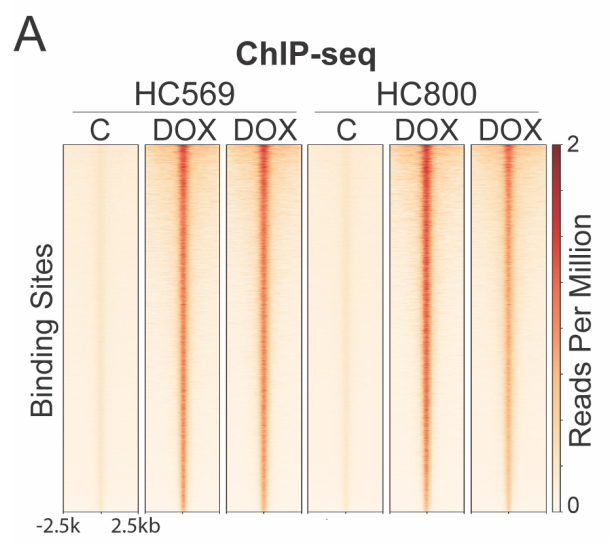

$\mathrm{D}$

\section{Differentially Expressed Genes}

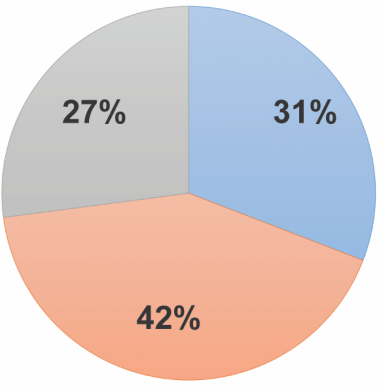

$\mathrm{F}$

\section{Chromatin accessibility}

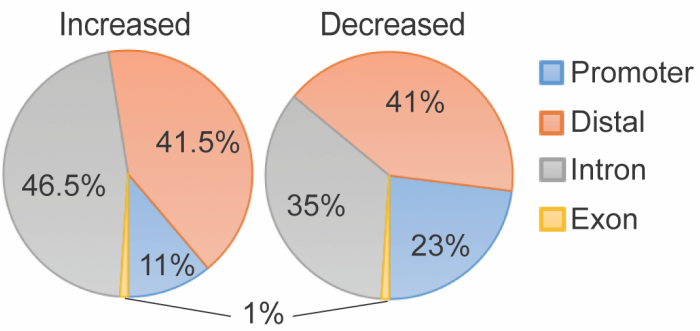

H

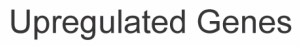

Repressed Genes
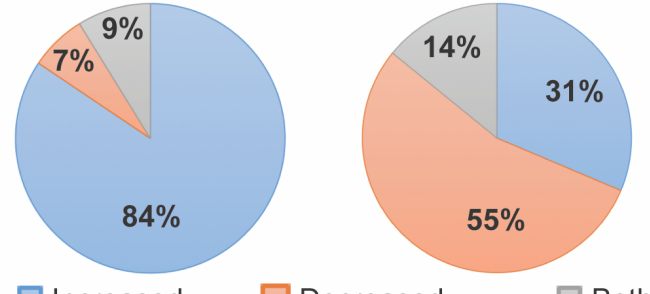

$\square$ Increased

Decreased

$\square$ Both

Chromatin accessibility

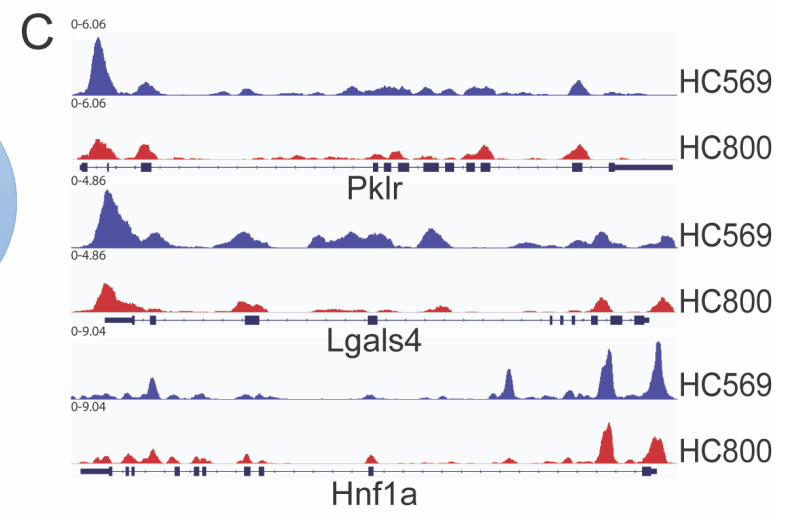

E

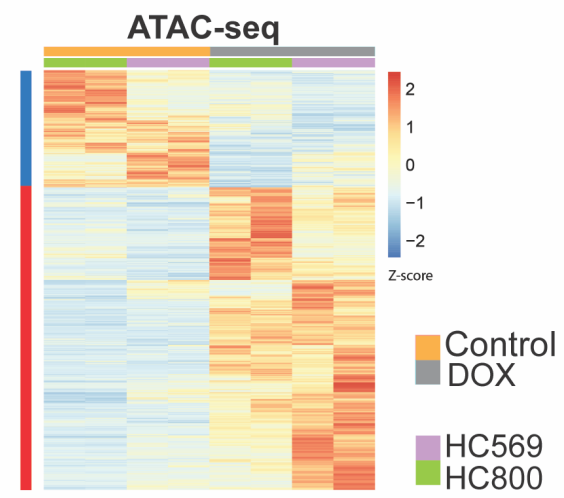

$G$
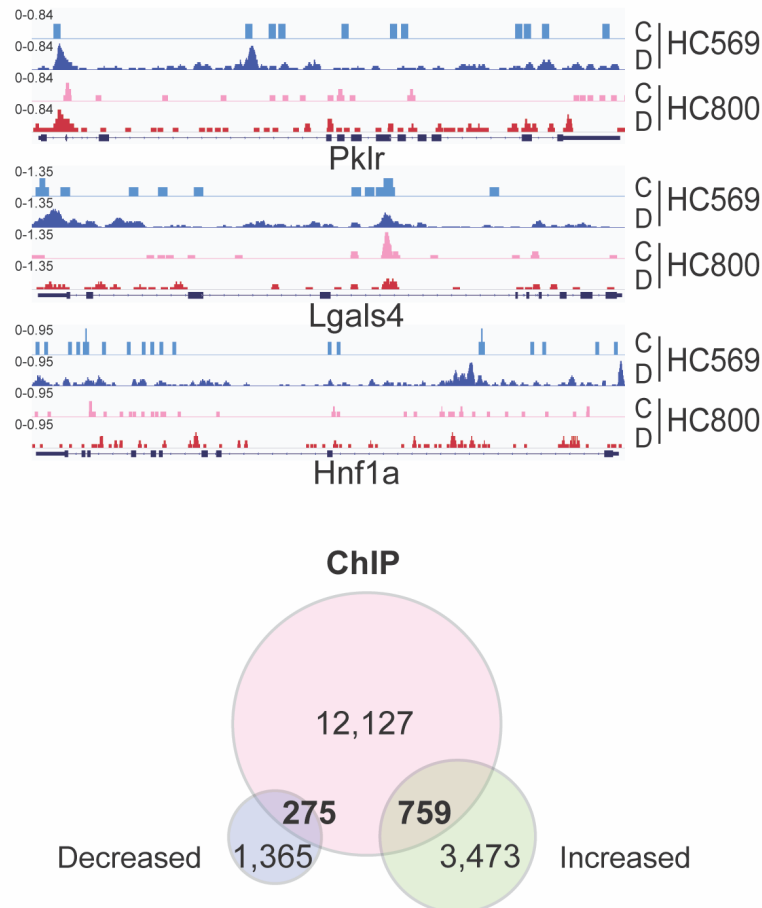

Chromatin accessibility 
Figure 5. Global analysis of HNF4 $\alpha$ occupancy and chromatin accessibility landscape in PDAC cells.

A. Heatmaps displaying $\mathrm{HNF} 4 \alpha$ binding sites in a 5-kb window around the ChIP-seq peak summits in HC569 and HC800 cells.

B. Pie chart displays the genomic distribution of HNF4 $\alpha$ binding sites.

C. Representative browser track images of three known direct targets of HNF4 $\alpha$ in HC569 (blue) and $\mathrm{HC} 800$ (red).

D. Overlap between RNA-seq and ChIP-seq data. Distribution of differentially expressed genes bound and not bound by HNF $4 \alpha$. Bound-UP: upregulated genes with HNF $4 \alpha$ occupancy. BoundDOWN: downregulated genes bound by HNF4 $\alpha$.

E. Heatmap shows signal at ATAC-seq regions that increase (red line) and decrease (blue line) chromatin accessibility upon dox-mediated HNF4 $\alpha$ induction in HC800 (green) and HC569 (purple) cell lines.

F. Distribution of genomic features for ATAC-seq regions with increased and reduced chromatin accessibility.

G. Representative browser track images showing intensity of ATAC-seq signal at the indicated genes in dox-treated cells (blue: HC569, red: HC800) compared to controls (light blue: HC569, pink: HC800) 
H. Overlap between RNA-seq and ATAC-seq data sets. Distribution of up- and downregulated genes between more and less accessible chromatin.

I. Venn diagram displays the overlap between HNF4 $\alpha$-bound sites and ATAC-seq peaks with increased or reduced genomic accessibility. 
We further assessed the effect of HNF4 $\alpha$ on global chromatin accessibility by performing ATACseq in both cell lines upon 1 week treatment with doxycycline or vehicle. Principal component analysis showed that control (HNF4 $\alpha$-negative) HC569 and HC800 cells clustered separately, but that HNF $4 \alpha$ restoration caused similar changes in chromatin accessibility in both cell lines (Figure S5C). A total of 5,872 significant ATAC-seq peaks were identified, with 4,232 sites exhibiting increased accessibility and 1,640 sites exhibiting decreased accessibility in the presence of HNF4 $\alpha$ (Figure 5E). Genomic distribution analysis of ATAC-seq peaks showed that most of the sites with significant changes in accessibility are found in distal regions and introns. Despite not being the most frequent genomic feature found in differentially accessible chromatin, promoters were overrepresented in closed genomic sites relative to chromatin with increased accessibility (Figure 5F). We observed increased signal of ATAC-seq peaks in HNF4 $\alpha$ target genes that are transcriptionally activated in dox-treated samples (Figure 5G). On further inspection, we observed that genomic regions with increased accessibility were enriched in motifs for binding of AP1 and HNF4 $\alpha$, among other nuclear receptor family members. In contrast, while closed chromatin peaks lack HNF4 $\alpha$ binding motifs, they show significant enrichment for AP1 binding sites as well (Figure S5D).

Intersection of ATAC-seq and RNA-seq data identified 743 differentially expressed genes (488 upregulated and 255 silenced genes) that displayed changes in chromatin accessibility upon HNF $4 \alpha$ restoration. The vast majority of the upregulated genes correlated with genomic regions with increased accessibility (84\%), while most of the repressed genes were associated with ATAC-seq peaks that had reduced genomic accessibility (55\%), although a considerable percentage $(31 \%)$ correlated with genomic areas that become more accessible (Figure $5 \mathrm{H}$ and S5E). 
Finally, correlation of HNF4 $\alpha$ binding sites with ATAC-seq data demonstrated that less than $10 \%$ of the HNF4 $\alpha$ bound sites displayed changes in chromatin accessibility and less than $20 \%$ of the ATAC-seq peaks with either more or less accessibility were occupied by HNF $4 \alpha$, suggesting that other transcription factors may be contributing to the HNF4 $\alpha$-dependent alterations in chromatin accessibility (Figure $5 \mathrm{I}$ and S5F).

Altogether, these results demonstrate that most of the differentially expressed genes that define the transcriptional network regulated by HNF4 $\alpha$ in PDAC constitute direct targets of this endodermal cell fate determinant. Additionally, our data suggest that most of the changes in chromatin accessibility seem to be indirect effects of HNF4 $\alpha$ restoration and not caused by its direct binding.

\section{HNF4 $\alpha$ inhibits the expression of mesodermal lineage specifiers SIX4 and SIX1 in PDAC}

Next, we sought to identify mechanisms by which loss of HNF $4 \alpha$ might lead to de-repression of a subset of genes associated with the Basal-like subtype. Six4, a member of the sine oculis homeobox family, is the most differentially expressed transcription factor in Hnf4a-deleted autochthonous tumors compared with controls (Figure 6A). Moreover, SIX4 expression is positively correlated with the Basal-like subtype of human PDAC and negatively correlated with Classical Score and HNF4A expression (Figure S6A). The Six4 paralogue Six1 is also differentially expressed in Hnf4a-deleted autochthonous tumors. Although SIX1 levels do not correlate with PDAC subtype to the same degree as SIX4 (Figure S6B), SIX1 levels positively correlate with SIX4 in human PDAC (Figure S6C). Overall, it appears that a subset of SIX4-high human PDAC also express high levels of SIX1 (Figure S6C). 


\section{Camolotto et al., Figure 6}

A
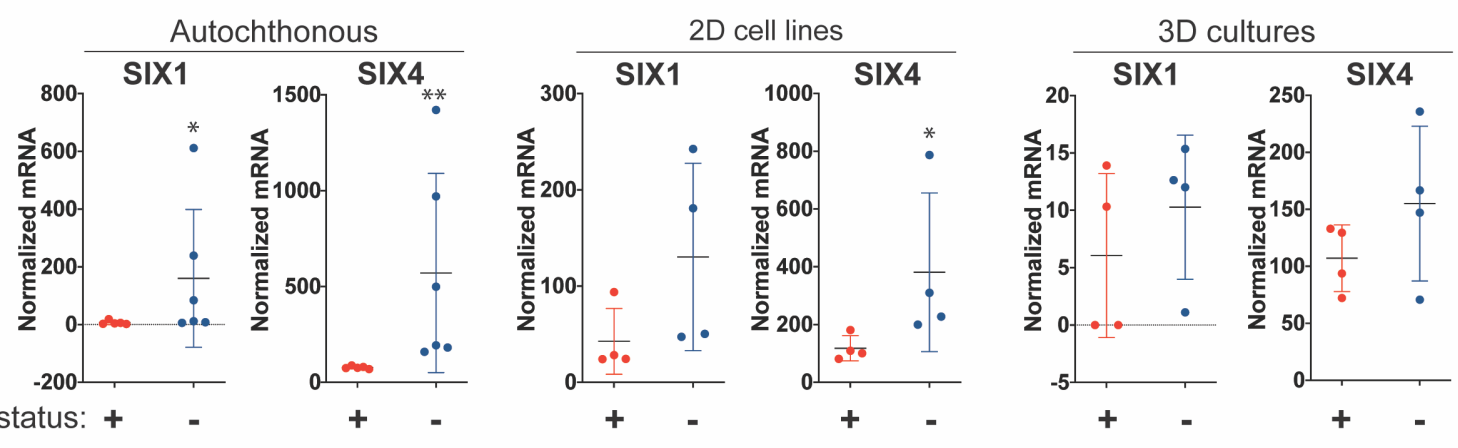

B

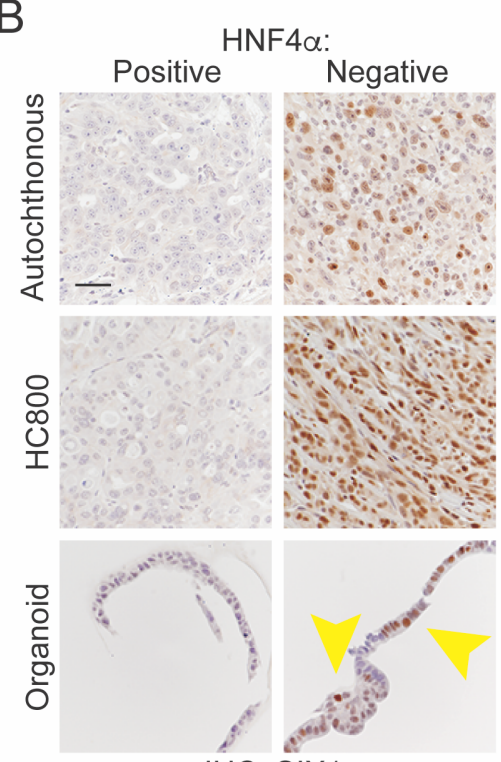

C

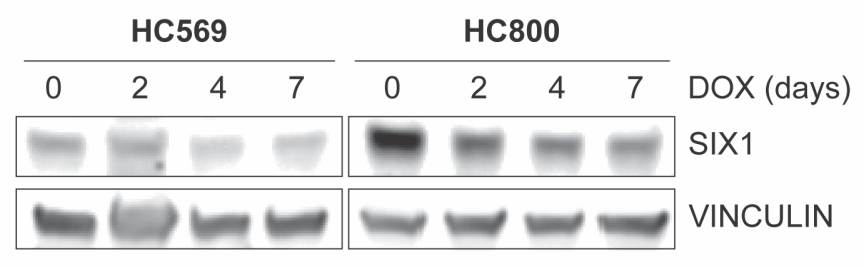

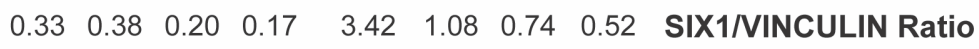

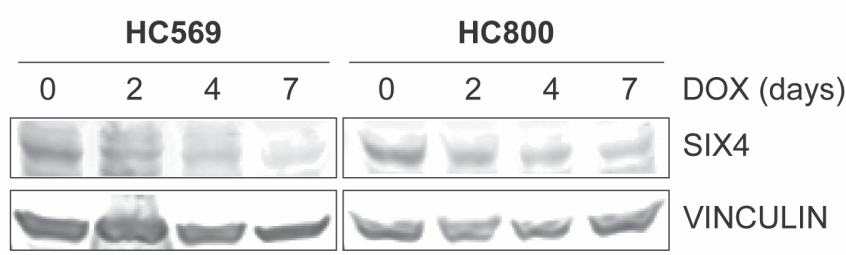

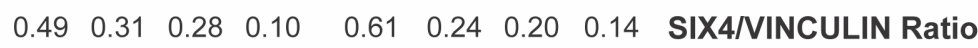

IHC: SIX1

D
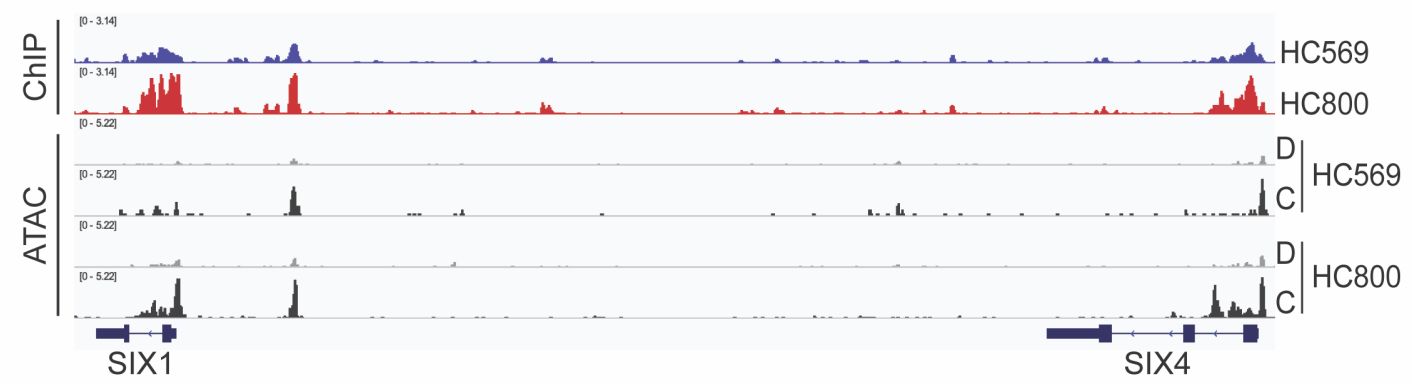
Figure 6. HNF4 $\alpha$ inhibits the expression of mesodermal lineage specifiers SIX1 and SIX4

A. Six1 and Six4 mRNA levels in indicated samples as assessed by RNA-Seq.

B. Representative IHC for SIX1 from indicated PDAC models with different HNF4 $\alpha$ expression levels. Autochthonous: Pdx1-Cre; Kras ${ }^{L S L-G 12 D} ; p 53^{F /+}$ tumors from Hnf4a ${ }^{+/+}$(left) or Hnf4a ${ }^{F / F}$ (right) mice. HC800: intraperitoneally injected cell line with dox-inducible HNF4 $\alpha$. Organoid: derived from Kras ${ }^{\text {FSF-G12D/+; }} p 53^{\text {Frt/Frt; }}$ Rosa26 $6^{\text {FSF-CreERT; }}$ Hnf4a $a^{\text {F/F }}$ mice. Three dimensional cultures were treated in vitro with vehicle control (left) or 4-hydroxy-tamoxifen (right) to delete Hnf4a. Yellow arrows indicate SIX1-positive cells. Scale bar: 100 microns.

C. Representative immunoblot for SIX1 (50ug) and SIX4 (100ug) in doxycycline- treated murine PDAC cell lines. Ratio between SIX1 or SIX4 and Vinculin (loading control) intensities are shown.

D. Browser track images display signal intensity of ChIP-Seq and ATAC-Seq in 2D lines.

Blue: HNF4 $\alpha$ ChIP in HC569. Red: HNF4 $\alpha$ ChIP in HC800. Grey: ATAC peaks in dox-treated cells. Black: ATAC signal in control cells. 
SIX4 and SIX1 are located immediately adjacent to each other in both mouse and human genomes and are co-expressed in several tissues during development (28). Both transcription factors bind the MEF3 DNA motif, and genetic knockout experiments have demonstrated their functional redundancy in the development of several mesodermally derived tissues, including skeletal muscle, sensory neurons, gonads and kidney (reviewed in (29)). SIX1 has been reported to promote the growth of the mesenchymal/Basal-like human PDAC cell lines Panc1 and MiaPaCa-2 $(30,31)$. However, there has been no systematic evaluation SIX4 in PDAC, either alone or in combination with SIX1, despite their co-expression in human tumors and evidence of significant functional redundancy during development.

To further investigate the ability of HNF4 $\alpha$ to regulate SIX1 and SIX4 expression, we evaluated RNA and protein levels in all of our mouse models. Analysis of RNA-Seq data from individual tumors showed that Six1 and Six4 were not expressed in $\mathrm{Hnf}_{4} \mathrm{a}^{+/+}$autochthonous tumors (Figure $6 \mathrm{~A}$, left). Three out of six $H n f 4 a^{F / F}$ tumors exhibited de-repression of both genes, whereas the other three $\mathrm{Hnf}_{4} \mathrm{a}^{\mathrm{F} / \mathrm{F}}$ tumors had very low levels of Six 1 and Six4 transcripts (similar to control tumors). We were able to evaluate SIX1 protein levels by IHC in an expanded panel of autochthonous tumors. We found that SIX1 was largely undetectable in $\mathrm{Hnf4a}^{+/+}$autochthonous tumors (Figure 6B, top row). In contrast, a panel of $H n f 4 a^{F / F}$ tumors from 12-week old mice $(n=14)$ exhibited a heterogenous pattern of SIX1 expression. SIX1 protein was detectable in 8 out of 14 $\mathrm{Hnf}_{4} \mathrm{a}^{\mathrm{F} / \mathrm{F}}$ tumors, whereas the other 6 tumors were negative. Within the 8 positive tumors, the percentage of SIX1-positive cells ranged from less than $5 \%$ to greater than $75 \%$ (Figure $6 \mathrm{~B}$, top row and data not shown). 
We next evaluated the ability of HNF4 $\alpha$ to regulate SIX1 and SIX4 in our other model systems. Exogenous HNF4 $\alpha$ significantly inhibited Six1/4 mRNA expression in both Hnf4a-deleted cell lines (HC569 and HC800) (Figure 6A, middle panels), which led to decreased SIX1 and SIX4 protein levels in vitro and correlated with virtually undetectable SIX1 expression in vivo (Figure 6B, middle row, and 6C). RNA-Seq analysis of organoids showed a non-significant trend toward increased Six4 levels after Hnf4a deletion (Figure 6A, right). Although Six1 did not score as a differentially expressed gene in organoids, we were able to detect differences at the protein level. Control murine PDAC organoids were uniformly SIX1-negative, but SIX1 protein was detectable in two of the four Hnf4a-deleted organoid lines (SC1640 and SC1853, Figure 6B, lower row). ChIP-seq revealed that HNF4 $\alpha$ directly binds both Six1 and Six4 genes in proximity to their promoter region in both cell lines. We also identified a significant peak in the intergenic region between Six1 and Six4. Moreover, HNF4 $\alpha$ occupancy in this region was accompanied by reduction in the chromatin accessibility (Figure 6D).

Taken together, these data show that HNF4 $\alpha$ represses SIX1 and SIX4, two lineage specifiers that drive mesodermal development, likely through direct binding to their regulatory elements. However, loss of HNF4 $\alpha$ expression is not sufficient for induction of SIX1 and SIX4 in all contexts, suggesting that other redundant mechanisms help repress SIX1/4 expression in the Classical subtype of PDAC.

\section{SIX1 and SIX4 are required for growth of HNF4 $\alpha$-negative PDAC in vitro and in vivo}

Given that Six genes are frequently dysregulated in different cancers, we decided to further explore the oncogenic function and potential redundancy of SIX1 and SIX4 in HNF4 $\alpha$-negative PDAC. We initially performed CRISPR-Cas9 genome editing to ablate Six1 and Six4 in HC569 and $\mathrm{HC} 800$ cells by using 2 independent single guide RNA sequences targeting Six 1 and 4 
bioRxiv preprint doi: https://doi.org/10.1101/814525; this version posted October 22, 2019. The copyright holder for this preprint (which was not certified by peer review) is the author/funder. All rights reserved. No reuse allowed without permission.

targeting Six4, which were validated by immunoblotting (Figure S7A). We used the three most efficient sgRNAs targeting Six4 for conducting functional studies. 


\section{Camolotto et al., Figure 7}

A

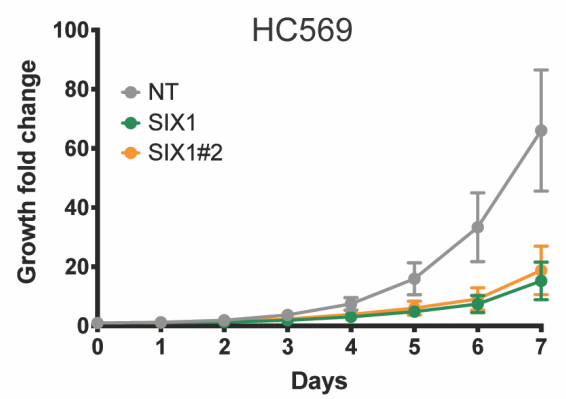

C

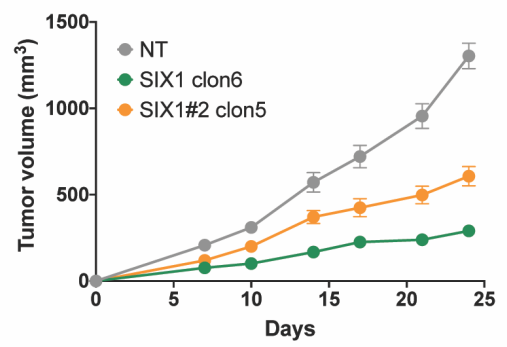

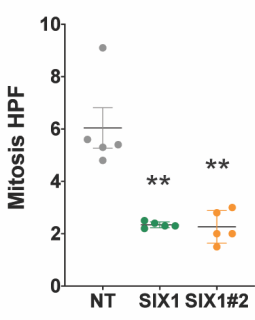

E

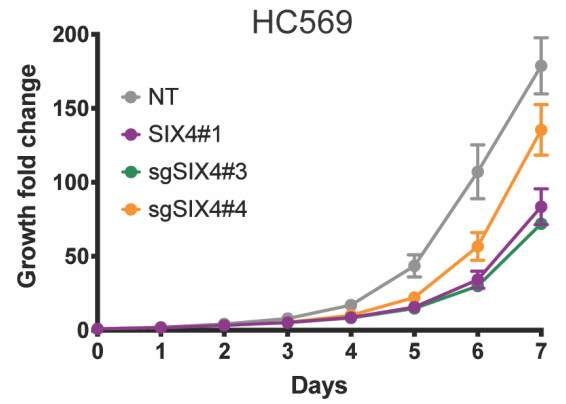

F

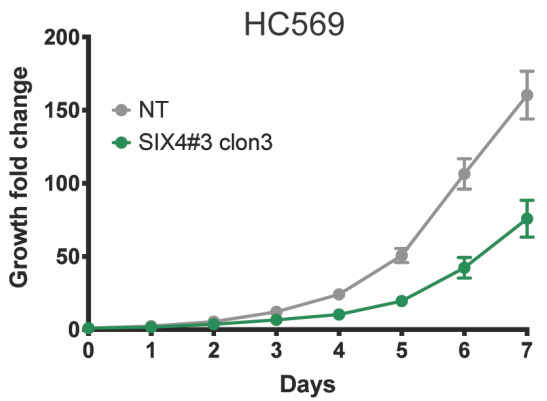

B

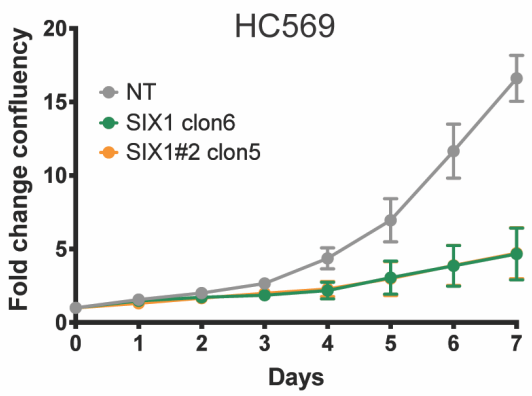

D
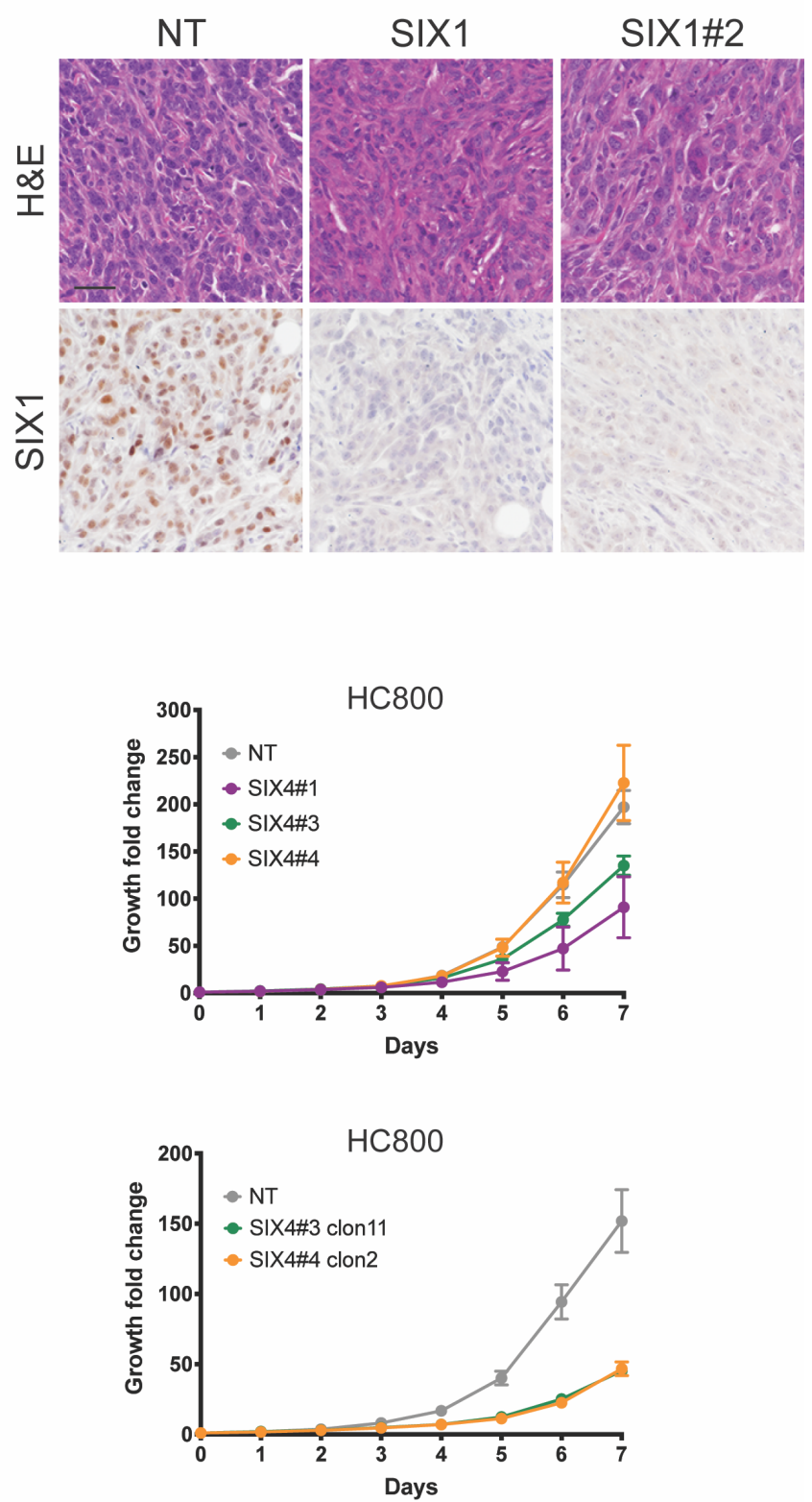
Figure 7. SIX4 and SIX1 are required for the growth of HNF4 $\alpha$-negative PDAC

A. Quantitation of proliferation in HC569 cells stably expressing Cas9 and the indicated sgRNA against Six1 ( $\mathrm{n}=3$ biological replicates, 2 independent experiments). Graph represents mean +/SEM. $p \leq 0.05$, Wilcoxon test.

B. Quantitation of proliferation in single cell cloned HC569 cells stably expressing Cas9 and the indicated sgRNA against Six1 ( $n=3$ biological replicates, 2 independent experiments). Data represents mean + - SEM. $p \leq 0.05$, Wilcoxon test.

C-D. Individual clonal populations of Six1 deleted-HC569 cell line were injected subcutaneously into the flank of NSG mice. Tumor volume was measured biweekly starting 1 week post implantation ( $n=5$ mice per group). C. Flank tumor volume and weight, and mitosis per high power field are shown. Data represented as mean \pm SEM. ${ }^{*} p<0.05 ;{ }^{* *} p<0.01 ;{ }^{* *} p<0.001$ by Wilcoxon test (for tumor growth) or Mann-Whitney test (for tumor weight and mitosis). D. Representative H\&E and IHC for SIX1 are shown for each cohort. Scale bar: 100 microns.

E. Quantitation of proliferation in HC800 and HC569 cells stably expressing Cas9 and the indicated sgRNA against Six4 ( $\mathrm{n}=3$ biological replicates, 2 independent experiments). Graph represents mean $+/$ - SEM. $p \leq 0.05$, Wilcoxon test.

F. Quantitation of proliferation in single cell cloned HC569 and HC800 cells stably expressing Cas9 and the indicated sgRNA targeting Six4 $(n=3$ biological replicates, 2 independent experiments). Graph represents mean +/- SEM. $p \leq 0.05$, Wilcoxon test. 
We first assessed the impact of SIX1 deletion in cell proliferation of the bulk population of both murine PDAC cell lines in vitro. HC569 cells stably transduced with either sgRNA against SIX1 showed a significant reduction in cell proliferation, indicating this cell line is susceptible to Six1 loss (Figure 7A). In contrast, loss of SIX1 expression in HC800 cells caused little to no change in proliferation relative to controls (Figure S7B). Single cell clones derived from these bulk populations revealed a similar pattern: in vitro proliferation of HC569 clones was remarkably impaired whereas HC800 clones showed almost no change (Figure 7B and S7C). In order to further explore the effect of Six1 loss in vivo, we injected single cell clones of each sgRNA into NSG mice. SIX1-negative clones formed tumors that were significantly smaller and less proliferative than controls (Figure $7 \mathrm{C}$ ). IHC confirmed the absence of SIX1 expression in tumors arising from both Six1-deleted clones, whereas SIX1 was readily detectable in control tumors (Figure 7D).

Six 4 knock out caused a reduction in the growth rates of both cell lines compared with control cells. In particular, HC569 showed a significant reduction in proliferation with all three sgRNAs, while only sgRNAs SIX4\#1 and \#3 caused a modest decrease in HC800 growth capacity (Figure 7E). We derived single cell clones of HC569 and HC800 SIX4 knock out cell lines and observed that SIX4-deleted cells displayed a slower growth compared to their control counterparts (Figure 7F).

Taken together, these results show that proliferation of both HNF4 $\alpha$-negative PDAC cell lines tested is dependent on SIX4 expression. One of the two lines tested is also highly dependent on SIX1 for both in vitro proliferation and in vivo tumorigenesis, consistent with prior reports in human cell lines $(30,31)$. 


\section{Discussion}

Although two major molecular subtypes of human PDAC have been characterized clinically, the molecular regulators of their distinct biologic properties and clinical behaviors have not been fully identified. Here we show that the nuclear receptor HNF4 $\alpha$ is a critical, non-redundant regulator of growth and molecular subtype in PDAC. Using complementary in vitro and in vivo model systems, we show that HNF4 $\alpha$ restrains tumor growth and enforces an epithelial differentiation program by at least two distinct mechanisms. First, HNF4 $\alpha$ directly activates multiple genes associated with the Classical subtype. HNF4 $\alpha$ targets include genes that play a direct role in aspects of epithelial cell biology, such as Lgals4, which encodes a member of the Galectin family that mediates intercellular adhesion and apical trafficking (32), tight junction components like claudin proteins, and desmosomal proteins such as DSC2 and DSG2. Moreover, HNF4 $\alpha$ activates other transcription factors (such as Hnf1a) that likely further amplify the transcriptional network governed by HNF4 $\alpha$. Second, HNF4 $\alpha$ directly represses the expression of Six 1 and Six4, genes encoding homeodomain transcription factors that are highly expressed in the Basallike subtype of PDAC and normally drive mesodermal development. Importantly, we find that HNF4 $\alpha$-negative PDAC cells are dependent on SIX4, and its close paralogue SIX1, for in vitro proliferation and in vivo tumorigenesis, suggesting that SIX4/1 activity may be a novel subtypespecific vulnerability in HNF4 $\alpha$-negative PDAC.

$\mathrm{HNF} 4 \alpha$ is likely one of several endodermal lineage specifiers that regulate the Classical molecular subtype of PDAC (reviewed in (33)). For example, GATA6, FoxA1 and FoxA2 have been reported to promote epithelial differentiation and block EMT in human PDAC cell lines $(34,35)$. Moreover, GATA6 binds and activates FOXA1 and FOXA2 (34), and FoxA1/2 activate HNF4A and other endodermal lineage specifiers in multiple contexts $(18,36)$. Based on these data, a potential hierarchy of endodermal lineage specifiers that regulate the Classical subtype of PDAC begins to 
emerge. However, these studies did not formally compare gene expression changes induced by manipulation of each transcription factor with the Classical and Basal-like transcriptome profiles. It will likely be necessary to modulate the expression of these transcriptional regulators within the same physiologically relevant model system(s) to gain a comprehensive understanding of their individual impact on specification of the Classical subtype as well as their hierarchical regulatory relationships, which may involve extensive cross-talk and feedback loops as in normal tissue (37).

The ability of HNF4 $\alpha$ to restrain tumor growth and overall progression of pancreatic tumors in our model systems is consistent with the better overall survival seen in the Classical subtype. Other endodermal cell fate determinants have been reported to restrain pancreatic cancer progression. For example, Ptf1a inhibits pancreatic tumorigenesis by not only preventing but also reversing tumor initiation (38). Nevertheless, it is clear the effect of endodermal lineage specifiers on tumor growth are to some degree context dependent. For example, an in vitro CRISPR screen identified $\mathrm{HNF} 4 \alpha$ (as well as its target HNF1 $\alpha$ ) as dependencies in two out of nine human PDAC cell lines tested (39). In this regard, dichotomous effects on PDAC growth have been reported for HNF1 $\alpha$ (40-42), PDX1 (43), FoxA1/2 (44-47) and GATA6 $(34,48,49)$. It seems likely that even though the most malignant subset of PDAC downregulates the endodermal differentiation program, loss of this program may only confer a selective advantage in specific situations or stages of tumor progression. Furthermore, a dichotomous role for lineage specifiers in regulating malignant potential appears to be an emerging principle in cancer biology. For example, the pulmonary lineage specifier NKX2-1 can function as an oncogene in EGFR-mutant lung adenocarcinoma but restrains the growth of KRAS-mutant lung adenocarcinoma (50). Moreover, HNF4 $\alpha$ itself is required for initiation and growth of NKX2-1-deficient lung adenocarcinoma, a subtype of lung cancer with a GI-like differentiation program similar to the Classical subtype of PDAC (18). 
The transcriptional network governing the Basal-like subtype of PDAC is even less well understood than the Classical subtype. The transcription factors $\Delta$ Np63 $(51,52)$ and Gli2 (53) have been reported to promote growth and activate a Basal-like gene expression program in human cell lines. Loss of the histone demethylase KDM6A can lead to de-repression of $\Delta$ Np63 and drive the development of squamous and poorly differentiated PDAC (54). Our work identifies the homeodomain transcription factors SIX4 and SIX1 as HNF4 $\alpha$-repressed drivers of proliferation and growth in the Basal-like subtype. HNF $4 \alpha$ binds directly to the promoters of Six4 and Six1 (as well as a potential distal regulatory element located between them), which is accompanied by decreased chromatin accessibility at these loci. The observed HNF4 $\alpha$ chromatin binding and genomic accessibility changes correlated with a marked decreased proliferative capacity of PDAC cells. SIX1 and SIX4 are partially redundant drivers of mesodermal differentiation in tissues such as skeletal muscle, sensory neurons, kidneys and gonads (29). Several SIX family members have been implicated as having oncogenic functions in various types of cancer $(55,56)$, including SIX1 in PDAC $(30,31)$. However, SIX4 has not been previously shown to play a functional role PDAC, despite its elevated levels in the Basal-like subtype. Intriguingly, both SIX4 and SIX1 physically interact with co-factors that are potentially druggable targets. SIX4 physically interacts with the lysine demethylase UTX/KDM6A, which can be targeted by small molecule inhibitors (57). Moreover, the EYA family of transcriptional co-activators are essential for SIX1 function and harbor a druggable Tyr phosphatase activity $(58,59)$. Thus, SIX4 and SIX1 may represent targetable vulnerabilities of the basal subtype of PDAC. Additional studies are necessary to understand the mechanistic role of these transcription factors in the malignant progression of PDAC.

Taken together, our findings demonstrate the role of HNF4 $\alpha$ as a regulator of a transcriptional network that restrains pancreatic cancer progression and imposes an epithelial cell identity and a 
Classical PDAC molecular subtype program that correlates with better outcome. Moreover, our results showed that $\mathrm{HNF} 4 \alpha$ directly represses SIX1 and SIX4, which play an oncogenic function in HNF4 $\alpha$-negative PDAC, leading to the identification of potentially relevant therapeutic vulnerabilities in this highly aggressive form of the disease.

\section{Material and Methods:}

\section{Mice}

Mice harboring Kras $^{L S L-G 12 D}$ (Jackson et al., 2001), Kras ${ }^{F S F-G 12 D}$ (Young et al., 2011), p53 flox $(60)$, $\operatorname{Rosa}^{\text {LSL-tdTomato (18), Hnf4a flox }}$ (14), Rosa-FSF-Cre ${ }^{E R T 2}$ (61), p53 fit (62), and Pdx1-Cre (19) alleles have been previously described. All animals were maintained on a mixed C57BL/6J x 129SvJ background. Animal studies were approved by the IACUC of the University of Utah and the University of California at San Francisco, and conducted in compliance with the Animal Welfare Act Regulations and other federal statutes relating to animals and experiments involving animals and adheres to the principles set forth in the Guide for the Care and Use of Laboratory Animals, National Research Council (PHS assurance registration number A-3031-01).

\section{Tamoxifen and Doxycycline Administration}

Mice were fed ad libitum food pellets supplemented with tamoxifen $(500 \mathrm{mg} / \mathrm{kg}$; Envigo, Indianopolis, IN) or doxycycline hyclate (625 mg/kg; Envigo, Indianopolis, IN) in place of standard chow starting 1 week before performing engraftments and for the entire duration of the experiments.

\section{Flank Tumor Transplantations}

For subcutaneous allo- and xenografts experiments, were injected with $2.5 \times 10^{5}$ murine PDAC 
cells, $3.5 \times 10^{5}$ human PDAC cells or $5 \times 10^{5}$ PDAC cells from organoid cultures were resuspended in $50 \mu \mathrm{L}$ of culture media, then mixed 1:1 with Matrigel (Corning). Cells were injected subcutaneously into the left or right flank NSG recipient mice. Subcutaneous tumor dimensions were measured with calipers twice weekly for the duration of the experiment, $n=8-10$ independent tumors per group. Tumor volume was calculated using the standard modified ellipsoid formula $1 / 2$ (Length $x$ Width2) formula. After completion of the experiment, tumors were removed, weighed, and fixed for histological analysis.

\section{In Vivo Bioluminescence Imaging}

Bioluminescence imaging was performed using an IVIS Spectrum In Vivo Imaging System (PerkinElmer). Mice bearing tumors of cell expressing firefly luciferase were injected with 200 $\mu \mathrm{l} /$ mouse of D-Luciferin (GoldBio) at $16.7 \mathrm{mg} / \mathrm{ml}$. Optimal signals were obtained $10 \mathrm{~min}$ after subcutaneous injections of the D-Luciferin. Scanning was done with mice placed in prone position.

\section{Histology and Immunohistochemistry}

All tissues were fixed in $10 \%$ formalin overnight and when necessary, lungs were perfused with formalin via the trachea. Organoids were first fixed in $10 \%$ formalin overnight and then mounted in HistoGel (Thermo Scientific, HG-4000-012). Mounted organoids and tissues were transferred to $70 \%$ ethanol, embedded in paraffin, and four-micrometer sections were cut. Immunohistochemistry (IHC) was performed manually on Sequenza slide staining racks (ThermoFisher Scientific, Waltham, MA). Sections were treated with Bloxall (Vector labs) followed by Horse serum 536 (Vector Labs, Burlingame, CA) or Rodent Block M (Biocare Medical, Pacheco, CA), primary antibody, and HRP-polymer-conjugated secondary antibody (anti-Rabbit, Goat and Rat from Vector Labs; anti-Mouse from Biocare. The slides were developed with Impact DAB (Vector) and counterstained with hematoxylin. Slides were stained with antibodies to BrdU (BU1/75, Abcam, Cambridge, MA) 1:400, HA tag (C29F4, CST) 1:400, Galectin 4 (AF2128, R\&D 
Systems) 1:200, HNF4 $\alpha$ (C11F12, CST) 1:500, PK-LR (EPR11093P, Abcam) 1:500, SIX1 (D5S2S, CST) 1:100, HNF4 $\alpha$ P1 and P2 (Human HNF-4 alpha/NR2A1 MAb (Clone K9218) PPK9218-00, Human HNF-4 alpha/NR2A1 MAb (Clone H6939) PP-H6939-00) 1:100. Pictures were taken on a Nikon Eclipse Ni-U microscope with a DS-Ri2 camera and NIS-Elements software. Mitoses quantitation and histological analyses were performed on hematoxylin and eosin-stained or IHC-stained slides using NIS-Elements software. All histopathologic analysis was performed by a board-certified anatomic pathologist (E.L.S.).

\section{Derivation of 2D Cell Lines and 3D Organoid Cultures}

Cell lines and 3D cultures were created by enzymatic and mechanical dissociation of pancreatic tumors or normal pancreatic tissue harvested from Kras ${ }^{L S L-G 12 D} ; p 53^{f l o x /+} ; H n f 4 a^{f l o x}$ flox; Pdx1-Cre and Kras ${ }^{F S F-G 12 D} ; p 53^{f / f f r t} ; H n f 4 a^{F / F} ;$ RosaCre ${ }^{E R T 2}$ mice, respectively. Two dimensional cultures were grown in DMEM supplemented with 10\%FBS. For organoid derivation, single cell suspensions were transduced with an adenovirus expressing Flp recombinase for 2 hs at $37^{\circ} \mathrm{C}$, washed, and seeded in grow factor-reduced Matrigel (Corning) and grown in 50\% L-WRN conditioned media (63). HNF4 $\alpha$ expression restoration was induced by supplementing the corresponding media with $1 \mathrm{ug} / \mathrm{ml}$ doxycycline (Sigma-Aldrich, D9891) and Hnf4a genetic loss was carried out by adding 4-OH-Tamoxifen (Cayman Chemical, 68392-35-8) to the culture media.

\section{Cell proliferation assay}

We used a live cell imaging system (IncuCyte) to directly measure cell proliferation while monitoring morphological changes over time. Number of cells was tracked by detecting the tdTomato present in HC569 and HC800 cell lines or by transducing cells to express eGFP fused to the histone $\mathrm{H} 2 \mathrm{~B}$, which labels cell nuclei. 


\section{Vector Cloning}

pCW-TRE-HNF4 $\alpha$ lentiviral vector was generated by PCR amplifying the HNF4 $\alpha$ isoform 8 cDNA from murine mucinous adenocarcinomas tumors and cloning into Hpal-Pacl sites of pCW22-TREcDNA;Ubc-rtTA plasmid. Correct identity and orientation of the construct was confirmed via Sanger sequencing.

\section{Lentiviral Production}

Lentivirus and retrovirus were produced by transfection of 293T cells with TransIT-293 (Mirus Bio). Packaging vectors $\Delta 8.9$ (gag/pol) and CMV-VSV-G (DuPage et al., 2009) were used for lentiviral production. Supernatant was collected at 48,60 and 72 hs post-transfection, centrifuged, and filtered using 0.45um filter units.

\section{cDNA Synthesis and qRT-PCR}

Quantitative RT-PCR was performed on Trizol-extracted RNA using the iScript Reverse Transcription Supermix (BIO-RAD). qPCR reactions were performed using SsoAdvanced Universal Probes Supermix (BIO-RAD) and a CFX384 Touch Real-Time PCR Detection System (BIO-RAD). The following Taqman probes were used: Hnf4a (Mm01247712_m1) and Pklr (Mm00443090_m1). Transcript levels were normalized to Ppia (Mm02342430_g1) and quantitated by the $\Delta \Delta \mathrm{Ct}$ method.

\section{Immunoblotting}

Cells were lysed on ice for 20 minutes in RIPA buffer (50mM Tris $\mathrm{HCl} \mathrm{pH} \mathrm{7.4,} 150 \mathrm{mM} \mathrm{NaCl}, 0.1$ $\%(\mathrm{w} / \mathrm{v})$ SDS, $0.5 \%(\mathrm{w} / \mathrm{v})$ sodium deoxycholate, $1 \%(\mathrm{v} / \mathrm{v})$ Triton X-100) plus Complete protease phosphatase inhibitor cocktail (A32961, ThermosFiher Scientific). Cells were pelleted for 10 minutes at $4{ }^{\circ} \mathrm{C}$ and protein concentration was quantitated with the Coomassie (Bradford) Protein Assay (ThermoFisher Scientific). Lysates were separated on Tris-Glycine (TGX) precast gels 
(Biorad) and transferred to nitrocellulose membranes (ThermoFisher Scientific). Membranes were probed with antibodies to HA tag (C29F4, CST, 1:5000), HNF4 $\alpha$ (C11F12, CST, 1:2000), ß-Tubulin (DSHB, 1:2000), Vinculin (Abcam, 1:20000), Six1 (D5S2S, CST, 1:1000), Six4 (D-5, Santa Cruz Biotechnology, 1:1000), and the appropriate species conjugated secondary antibodies (ThermoFisher Scientific, 1:20000), and visualized using an Odyssey CLx Imaging System (LI-COR Biosciences).

\section{Chromatin Immunoprecipitation (ChIP-seq) Assay and Analysis}

After treating HC569 and HC800 with DOX or vehicle for 1 week, cells were crosslinked with $1 \%$ formaldehyde for $10 \mathrm{~min}$ at room temperature and then treated with $125 \mathrm{mM}$ glycine for $5 \mathrm{~min}$ to stop crosslinking. Crosslinked cells were then washed with cold PBS and scraped to harvest. ChIP was performed as previously described (Reddy et al., 2009). Sonication was performed on Active Motif EpiShear Probe Sonicator with 8 cycles of 30 s of amplitude $40 \%$ sonication and 30 $\mathrm{S}$ of rest. The antibody used was the purified anti-HA.11 Epitope Tag Antibody (16B12, BioLegend). Reads were aligned to the $\mathrm{mm} 10$ build of the mouse genome using Bowtie with the following parameters: -m 1 -t-best -q -S -I 32 -e 80 -n 2 (64). Peaks were called using ModelBased Analysis of ChIP-seq-2 (MACS2) (Zhang et al., 2008) with a p value cutoff of $1 \mathrm{e}-10$ and the mfold parameter constrained between 15 and 100. ChIP-seq peaks that were replicated within each cell line were used for downstream analysis. Motif finding was performed on $100 \mathrm{bp}$ surrounding the top 1000 peaks based on their integer score (column 5 of narrowPeak file). Motifs were discovered using the meme suite (Bailey et al., 2009), searching for motifs between 6 and 50 bases in length, with zero or one occurrence per sequence. Genes were assigned to ChIP. seq peaks using GREAT with a 100kb distance cutoff (65).

\section{Assay for Transposase Accessible Chromatin (ATAC-seq) and Analysis}


Following doxycycline treatment for 1 weeks, ATAC-seq was performed on 250,000 cells for each library (described by Buenrostro et al., 2013). Tn5 transposase, with Illumina adapters, was constructed as outlined earlier (Picelli et al., 2014). Sequencing reads were aligned to mm10 using Bowtie (Langmead et al., 2009) with the following parameters: -m 1 -t-best -q -S -l 32 -e 80 -n 2. SAM files were converted to BAM files and sorted using samtools (Li et al., 2009). MACS2 (Zhang et al., 2008) was used to call peaks without a control input. We used a cutoff p-value of 1e-10 when calling peaks. Feature Counts (Liao et al., 2014) was used to quantify reads that aligned in regions \pm 250 bp Hnf $4 \alpha$-binding site summits from the ChIP-seq experiments. These reads were then normalized and differential expression was determined by comparing samples in a pairwise manner using the DESeq2 package for $\mathrm{R}$ (Love et al., 2014). DESeq2 was used with a multivariate model that corrected for cell line and then identified significant effects from doxycycline treatment. Genes were assigned to ATAC-seq peaks using GREAT with a 100kb distance cutoff (65).

\section{RNA sequencing}

RNA was isolated from aniline blue-stained FFPE sections of micro-dissected autochthonous tumors by the Molecular Diagnostic core facility $(\mathrm{HCl})$ using the miRNAeasy kit (Qiagen). IHC for HNF $4 \alpha$ on serial sections was used to identify viable tumor areas positive or negative for HNF $4 \alpha$. RNA was isolated from cell lines and organoid cultures by Trizol-chloroform extraction followed by column-based purification. The aqueous phase was brought to a final concentration of $50 \%$ ethanol, and RNA was purified using the PureLink RNA Mini kit according to the manufacturer's instructions (ThermoFisher Scientific). Library preparation was performed using the TruSeq Stranded RNA kit with Ribo-Zero Gold (Illumina). Libraries from FFPE purified samples were sequenced on an Illumina HiSeq 2500 (50 cycle single-read sequencing) and samples from 2D cells and organoids were sequenced on a NovaSeq 6000 (50bp paired-end sequencing). 


\section{RNA-seq Data Processing and Analysis}

Mouse FASTA and GTF files were downloaded from Ensembl release 92 and a reference database was created using using STAR version 2.5.4a (66)) with splice junctions optimized for 50 base pair reads. Reads were trimmed of adapters using cutadapt 1.16 (67) and then aligned

to the reference database using STAR in two pass mode to output a BAM file sorted by coordinates. Mapped reads were assigned to annotated genes in the GTF file using featureCounts version 1.6.3 (68). The gene counts were filtered to remove 18,611 features with zero counts and 12,997 features with fewer than 5 reads in every sample. Differentially expressed genes were identified using a 5\% false discovery rate with DESeq2 version 1.22 .2 (69).

\section{Comparison of RNA-seq Data with Public Data}

TCGA survival data was downloaded from Supplemental Table 1 of (5) while the mRNA expression data was downloaded using the R package TCGABiolinks. The cohort was trimmed to only include samples of high purity confirmed adenocarcinomas ( $>30 \%$ tumor, $n=76$ ), and then was further subdivided by HNF4A mRNA expression. The PACA-AU data and associated metadata were downloaded from the icgc.org data portal, with the supplemental data coming from (2). This study excluded samples with $<40 \%$ purity, so no further trimming was necessary.

For generation of basal-like and classical scores of mouse tumors, the human gene signatures from Moffitt et al. were used ( $n=25$ per subtype). Only genes with identified mouse homologs were considered (basal-like $n=14$, classical $n=18$ ). Expression was log normalized, and the mean expression of all gene set members was defined as the overall expression score. For gene set enrichment, expression data was split into two conditions (Hnf4a+ and Hnf4a-) and a rank order list prepared per gene using the difference in means divided by the sum of standard deviations for samples from each condition.

For presentation of genes that are differentially expressed across model systems (Supplemental 
Table S5), "up" genes includes all three datasets, whereas "down" genes includes only autochthonous tumors and 2D cell lines because there was little overlap between "down" genes in the organoid system in comparison with the other models.

\section{CRISPR-Cas9 Genome Editing}

SIX1 and SIX4 knock-out cell lines were generated by CRISPR-Cas9. LentiCRISPRv2 vectors, which express the single guide RNA (sgRNA), Cas9 and a selection cassette, were obtained from Addgene (ID: 52961 and 98291).

Cells were transduced and then subjected to puromycin or hygromycin selection, as corresponds. Two different sgRNA sequences were used to target Six1, four were tested for Six4, and an inert non-targeting guide was used as a control.

sgSIX1 GTGGCTGAAAGCGCACTACG (Reference: (31)).

sgSIX1\#2 GGAGGGAACCTGGAACGCCT

sgSIX4\#1 AAGTGCGGCGGACATCAAGC

sgSIX4\#2 CGCGGCGTCCCCCGGCTCCA

sgSIX4\#3 GCGCACCGAGAAGTGGCGGG

sgSIX4\#4 TGCCTCCAGGGTAAGCCGGG

sgNT GCGAGGTATTCGGCTCCGCG (Reference: (70)).

Individual cells were allowed to expand for a period of 3-6 weeks, after which time clonal expansions of Six1 knock outs grew. Confirmation of efficient deletion was carried out by detecting protein loss by Immunoblot for endogenous SIX1 and exogenously over-expressed SIX4, as its endogenous levels were essentially undetectable due to lack of antibody sensitivity.

\section{Statistics}


Statistical analyses were carried out using Prism 8. p-Values were calculated using MannWhitney $U$ test, Chi-square test, Log-rank test or Wilcoxon test. RNA-Seq statistics are described above.

\section{Data availability}

Gene expression, ChIP-Seq and ATAC-seq data are accessible in the NCBI Gene Expression Omnibus (GEO) database under accession number GSE138145 and GSE138463.

\section{Acknowledgements}

We are grateful to members of the Snyder lab for suggestions and comments. We thank Brian Dalley for sequencing expertise and James Marvin for FACS expertise. Core facilities (PRR, BMP, DNA sequencing, Genomics/Bioinformatics, Flow Cytometry). Research reported in this publication utilized shared resources (including Flow Cytometry, High Throughput Genomics, Bioinformatics, and Biorepository and Molecular Pathology) at the University of Utah and was supported by the National Cancer Institute of the National Institutes of Health under Award Number 5R21CA194764-03. Work in the flow cytometry core was also supported by the National Center for Research Resources of the National Institutes of Health under Award Number 1S20RR026802-1. ELS was supported in part by a Career Award for Medical Scientists from the Burroughs Wellcome Fund, a V Scholar Award, the NIH (R01CA212415) and institutional funds (Department of Pathology and Huntsman Cancer Institute, University of Utah).

Author contributions: SC and ES designed experiments. SC, VB, KB and HC performed experiments. SC, LTH, JV, CS, RM, JG and ES analyzed data. ES performed histopathologic review. SC, and ES wrote the manuscript. All authors discussed results, reviewed and revised the manuscript. 


\section{References}

1. Maitra A, Hruban RH. Pancreatic cancer. Annu Rev Pathol 2008;3:157-88

2. Bailey P, Chang DK, Nones K, Johns AL, Patch AM, Gingras MC, et al. Genomic analyses identify molecular subtypes of pancreatic cancer. Nature 2016;531:47-52

3. Collisson EA, Sadanandam A, Olson P, Gibb WJ, Truitt M, Gu S, et al. Subtypes of pancreatic ductal adenocarcinoma and their differing responses to therapy. Nat Med $2011 ; 17: 500-3$

4. Moffitt RA, Marayati R, Flate EL, Volmar KE, Loeza SG, Hoadley KA, et al. Virtual microdissection identifies distinct tumor- and stroma-specific subtypes of pancreatic ductal adenocarcinoma. Nat Genet 2015;47:1168-78

5. TCGA. Integrated Genomic Characterization of Pancreatic Ductal Adenocarcinoma. Cancer Cell 2017;32:185-203 e13

6. Aung KL, Fischer SE, Denroche RE, Jang GH, Dodd A, Creighton S, et al. GenomicsDriven Precision Medicine for Advanced Pancreatic Cancer: Early Results from the COMPASS Trial. Clin Cancer Res 2018;24:1344-54

7. Prasad NB, Biankin AV, Fukushima N, Maitra A, Dhara S, Elkahloun AG, et al. Gene expression profiles in pancreatic intraepithelial neoplasia reflect the effects of Hedgehog signaling on pancreatic ductal epithelial cells. Cancer research 2005;65:1619-26

8. Tanaka M, Shibahara J, Fukushima N, Shinozaki A, Umeda M, Ishikawa S, et al. Claudin18 is an early-stage marker of pancreatic carcinogenesis. The journal of histochemistry and cytochemistry : official journal of the Histochemistry Society 2011;59:942-52

9. Cattin AL, Le Beyec J, Barreau F, Saint-Just S, Houllier A, Gonzalez FJ, et al. Hepatocyte nuclear factor 4alpha, a key factor for homeostasis, cell architecture, and barrier function of the adult intestinal epithelium. Molecular and cellular biology 2009;29:6294-308 
10. Moore BD, Khurana SS, Huh WJ, Mills JC. Hepatocyte nuclear factor 4alpha is required for cell differentiation and homeostasis in the adult mouse gastric epithelium. American journal of physiology Gastrointestinal and liver physiology 2016;311:G267-75

11. Santangelo L, Marchetti A, Cicchini C, Conigliaro A, Conti B, Mancone C, et al. The stable repression of mesenchymal program is required for hepatocyte identity: a novel role for hepatocyte nuclear factor 4alpha. Hepatology 2011;53:2063-74

12. Sekiya S, Suzuki A. Direct conversion of mouse fibroblasts to hepatocyte-like cells by defined factors. Nature 2011;475:390-3

13. Bonzo JA, Ferry CH, Matsubara T, Kim JH, Gonzalez FJ. Suppression of Hepatocyte Proliferation by Hepatocyte Nuclear Factor 4alpha in Adult Mice. The Journal of biological chemistry 2012

14. Hayhurst GP, Lee YH, Lambert G, Ward JM, Gonzalez FJ. Hepatocyte nuclear factor 4alpha (nuclear receptor 2A1) is essential for maintenance of hepatic gene expression and lipid homeostasis. Mol Cell Biol 2001;21:1393-403

15. Hatziapostolou M, Polytarchou C, Aggelidou E, Drakaki A, Poultsides GA, Jaeger SA, et al. An HNF4alpha-miRNA inflammatory feedback circuit regulates hepatocellular oncogenesis. Cell 2011;147:1233-47

16. Kim J, Hoffman JP, Alpaugh RK, Rhim AD, Reichert M, Stanger BZ, et al. An iPSC line from human pancreatic ductal adenocarcinoma undergoes early to invasive stages of pancreatic cancer progression. Cell Rep 2013;3:2088-99

17. Taguchi A, Politi K, Pitteri SJ, Lockwood WW, Faca VM, Kelly-Spratt K, et al. Lung cancer signatures in plasma based on proteome profiling of mouse tumor models. Cancer Cell 2011;20:289-99

18. Snyder EL, Watanabe H, Magendantz M, Hoersch S, Chen TA, Wang DG, et al. Nkx2-1 represses a latent gastric differentiation program in lung adenocarcinoma. Molecular cell 2013;50:185-99 
19. Hingorani SR, Petricoin EF, Maitra A, Rajapakse V, King C, Jacobetz MA, et al. Preinvasive and invasive ductal pancreatic cancer and its early detection in the mouse. Cancer Cell 2003;4:437-50

20. Perez-Mancera PA, Guerra C, Barbacid M, Tuveson DA. What we have learned about pancreatic cancer from mouse models. Gastroenterology 2012;142:1079-92

21. Rozenblum E, Schutte M, Goggins M, Hahn SA, Panzer S, Zahurak M, et al. Tumorsuppressive pathways in pancreatic carcinoma. Cancer Res 1997;57:1731-4

22. Karnoub AE, Weinberg RA. Ras oncogenes: split personalities. Nat Rev Mol Cell Biol 2008;9:517-31

23. Ko HL, Zhuo Z, Ren EC. HNF4alpha Combinatorial Isoform Heterodimers Activate Distinct Gene Targets that Differ from Their Corresponding Homodimers. Cell Rep 2019;26:254957.e3

24. Chellappa K, Deol P, Evans JR, Vuong LM, Chen G, Briancon N, et al. Opposing roles of nuclear receptor HNF4alpha isoforms in colitis and colitis-associated colon cancer. Elife 2016;5

25. Vuong LM, Chellappa K, Dhahbi JM, Deans JR, Fang B, Bolotin E, et al. Differential Effects of Hepatocyte Nuclear Factor 4alpha Isoforms on Tumor Growth and T-Cell Factor 4/AP1 Interactions in Human Colorectal Cancer Cells. Mol Cell Biol 2015;35:3471-90

26. Kinsey CG, Camolotto SA, Boespflug AM, Guillen KP, Foth M, Truong A, et al. Protective autophagy elicited by RAF-->MEK-->ERK inhibition suggests a treatment strategy for RAS-driven cancers. Nat Med 2019;25:620-7

27. Wollny D, Zhao S, Everlien I, Lun X, Brunken J, Brune D, et al. Single-Cell Analysis Uncovers Clonal Acinar Cell Heterogeneity in the Adult Pancreas. Dev Cell 2016;39:289301

28. Kumar JP. The sine oculis homeobox (SIX) family of transcription factors as regulators of development and disease. Cell Mol Life Sci 2009;66:565-83 
29. Fujimoto $\mathrm{Y}$, Tanaka SS, Yamaguchi YL, Kobayashi H, Kuroki S, Tachibana M, et al. Homeoproteins Six1 and Six4 regulate male sex determination and mouse gonadal development. Dev Cell 2013;26:416-30

30. Lerbs T, Bisht S, Scholch S, Pecqueux M, Kristiansen G, Schneider M, et al. Inhibition of Six1 affects tumour invasion and the expression of cancer stem cell markers in pancreatic cancer. BMC Cancer 2017;17:249

31. Li Z, Tian T, Lv F, Chang Y, Wang X, Zhang L, et al. Six1 promotes proliferation of pancreatic cancer cells via upregulation of cyclin D1 expression. PLoS One 2013;8:e59203

32. Cao ZQ, Guo XL. The role of galectin-4 in physiology and diseases. Protein Cell $2016 ; 7: 314-24$

33. Camolotto SA, Belova VK, Snyder EL. The role of lineage specifiers in pancreatic ductal adenocarcinoma. J Gastrointest Oncol 2018;9:1005-13

34. Martinelli P, Carrillo-de Santa Pau E, Cox T, Sainz B, Jr., Dusetti N, Greenhalf W, et al. GATA6 regulates EMT and tumour dissemination, and is a marker of response to adjuvant chemotherapy in pancreatic cancer. Gut 2017;66:1665-76

35. Song $\mathrm{Y}$, Washington MK, Crawford HC. Loss of FOXA1/2 is essential for the epithelial-tomesenchymal transition in pancreatic cancer. Cancer Res 2010;70:2115-25

36. Duncan SA, Navas MA, Dufort D, Rossant J, Stoffel M. Regulation of a transcription factor network required for differentiation and metabolism. Science 1998;281:692-5

37. Lau HH, Ng NHJ, Loo LSW, Jasmen JB, Teo AKK. The molecular functions of hepatocyte nuclear factors - In and beyond the liver. J Hepatol 2018;68:1033-48

38. Krah NM, Narayanan SM, Yugawa DE, Straley JA, Wright CVE, MacDonald RJ, et al. Prevention and Reversion of Pancreatic Tumorigenesis through a Differentiation-Based Mechanism. Dev Cell 2019;50:744-54.e4 
39. Lu B, Klingbeil O, Tarumoto $\mathrm{Y}$, Somerville TDD, Huang $\mathrm{YH}$, Wei $\mathrm{Y}$, et al. A Transcription Factor Addiction in Leukemia Imposed by the MLL Promoter Sequence. Cancer Cell 2018;34:970-81.e8

40. Abel EV, Goto M, Magnuson B, Abraham S, Ramanathan N, Hotaling E, et al. HNF1A is a novel oncogene that regulates human pancreatic cancer stem cell properties. Elife 2018;7

41. Hoskins JW, Jia J, Flandez M, Parikh $\mathrm{H}$, Xiao W, Collins I, et al. Transcriptome analysis of pancreatic cancer reveals a tumor suppressor function for HNF1A. Carcinogenesis 2014;35:2670-8

42. Luo Z, Li Y, Wang H, Fleming J, Li M, Kang Y, et al. Hepatocyte nuclear factor 1A (HNF1A) as a possible tumor suppressor in pancreatic cancer. PLoS One 2015;10:e0121082

43. Roy N, Takeuchi KK, Ruggeri JM, Bailey P, Chang D, Li J, et al. PDX1 dynamically regulates pancreatic ductal adenocarcinoma initiation and maintenance. Genes Dev 2016;30:2669-83

44. Diaferia GR, Balestrieri C, Prosperini E, Nicoli P, Spaggiari P, Zerbi A, et al. Dissection of transcriptional and cis-regulatory control of differentiation in human pancreatic cancer. Embo j 2016;35:595-617

45. Kondratyeva LG, Sveshnikova AA, Grankina EV, Chernov IP, Kopantseva MR, Kopantzev EP, et al. Downregulation of expression of mater genes SOX9, FOXA2, and GATA4 in pancreatic cancer cells stimulated with TGFbeta1 epithelial-mesenchymal transition. Dokl Biochem Biophys 2016;469:257-9

46. Roe JS, Hwang CI, Somerville TDD, Milazzo JP, Lee EJ, Da Silva B, et al. Enhancer Reprogramming Promotes Pancreatic Cancer Metastasis. Cell 2017;170:875-88 e20

47. Vorvis C, Hatziapostolou M, Mahurkar-Joshi S, Koutsioumpa M, Williams J, Donahue TR, et al. Transcriptomic and CRISPR/Cas9 technologies reveal FOXA2 as a tumor 
suppressor gene in pancreatic cancer. American journal of physiology Gastrointestinal and liver physiology 2016;310:G1124-37

48. Kwei KA, Bashyam MD, Kao J, Ratheesh R, Reddy EC, Kim YH, et al. Genomic profiling identifies GATA6 as a candidate oncogene amplified in pancreatobiliary cancer. PLoS Genet 2008;4:e1000081

49. Zhong Y, Wang Z, Fu B, Pan F, Yachida S, Dhara M, et al. GATA6 activates Wnt signaling in pancreatic cancer by negatively regulating the Wnt antagonist Dickkopf-1. PLoS One 2011;6:e22129

50. Skoulidis F, Heymach JV. Co-occurring genomic alterations in non-small-cell lung cancer biology and therapy. Nature reviews Cancer 2019;19:495-509

51. Hamdan FH, Johnsen SA. DeltaNp63-dependent super enhancers define molecular identity in pancreatic cancer by an interconnected transcription factor network. Proc Natl Acad Sci U S A 2018;115:E12343-e52

52. Somerville TDD, Xu Y, Miyabayashi K, Tiriac H, Cleary CR, Maia-Silva D, et al. TP63Mediated Enhancer Reprogramming Drives the Squamous Subtype of Pancreatic Ductal Adenocarcinoma. Cell Rep 2018;25:1741-55.e7

53. Adams CR, Htwe HH, Marsh T, Wang AL, Montoya ML, Subbaraj L, et al. Transcriptional control of subtype switching ensures adaptation and growth of pancreatic cancer. Elife 2019;8

54. Andricovich J, Perkail S, Kai Y, Casasanta N, Peng W, Tzatsos A. Loss of KDM6A Activates Super-Enhancers to Induce Gender-Specific Squamous-like Pancreatic Cancer and Confers Sensitivity to BET Inhibitors. Cancer Cell 2018;33:512-26.e8

55. Armat M, Ramezani F, Molavi O, Sabzichi M, Samadi N. Six family of homeobox genes and related mechanisms in tumorigenesis protocols. Tumori 2016;2016:236-43 
56. Tang X, Yang Y, Song X, Liu X, Wang X, Huang F, et al. SIX4 acts as a master regulator of oncogenes that promotes tumorigenesis in non-small-cell lung cancer cells. Biochemical and biophysical research communications 2019;516:851-7

57. Chakroun I, Yang D, Girgis J, Gunasekharan A, Phenix H, Kaern M, et al. Genome-wide association between Six4, MyoD, and the histone demethylase Utx during myogenesis. FASEB journal : official publication of the Federation of American Societies for Experimental Biology 2015;29:4738-55

58. Anantharajan J, Zhou H, Zhang L, Hotz T, Vincent MY, Blevins MA, et al. Structural and Functional Analyses of an Allosteric EYA2 Phosphatase Inhibitor That Has On-Target Effects in Human Lung Cancer Cells. Mol Cancer Ther 2019;18:1484-96

59. Blevins MA, Towers CG, Patrick AN, Zhao R, Ford HL. The SIX1-EYA transcriptional complex as a therapeutic target in cancer. Expert opinion on therapeutic targets 2015;19:213-25

60. Jonkers J, Meuwissen R, van der Gulden $H$, Peterse $H$, van der Valk M, Berns A. Synergistic tumor suppressor activity of BRCA2 and p53 in a conditional mouse model for breast cancer. Nature genetics 2001;29:418-25

61. Schonhuber N, Seidler B, Schuck K, Veltkamp C, Schachtler C, Zukowska M, et al. A nextgeneration dual-recombinase system for time- and host-specific targeting of pancreatic cancer. Nat Med 2014;20:1340-7

62. Lee CL, Moding EJ, Huang X, Li Y, Woodlief LZ, Rodrigues RC, et al. Generation of primary tumors with Flp recombinase in FRT-flanked p53 mice. Disease models \& mechanisms 2012;5:397-402

63. Miyoshi H, Stappenbeck TS. In vitro expansion and genetic modification of gastrointestinal stem cells in spheroid culture. Nat Protoc 2013;8:2471-82

64. Langmead B, Trapnell C, Pop M, Salzberg SL. Ultrafast and memory-efficient alignment of short DNA sequences to the human genome. Genome biology 2009;10:R25 
65. McLean CY, Bristor D, Hiller M, Clarke SL, Schaar BT, Lowe CB, et al. GREAT improves functional interpretation of cis-regulatory regions. Nat Biotechnol 2010;28:495-501

66. Dobin A, Davis CA, Schlesinger F, Drenkow J, Zaleski C, Jha S, et al. STAR: ultrafast universal RNA-seq aligner. Bioinformatics 2013;29:15-21

67. Martin M. Cutadapt removes adapter sequences from high-throughput sequencing reads. EMBnetjournal 2011;17

68. Liao Y, Smyth GK, Shi W. featureCounts: an efficient general purpose program for assigning sequence reads to genomic features. Bioinformatics 2014;30:923-30

69. Love MI, Huber W, Anders S. Moderated estimation of fold change and dispersion for RNA-seq data with DESeq2. Genome Biol 2014;15:550

70. Chiou SH, Winters IP, Wang J, Naranjo S, Dudgeon C, Tamburini FB, et al. Pancreatic cancer modeling using retrograde viral vector delivery and in vivo CRISPR/Cas9-mediated somatic genome editing. Genes Dev 2015;29:1576-85 